\title{
Quenched invariance principles for the discrete Fourier transforms of a stationary process
}

\author{
DAVID BARRERA ${ }^{1,2}$ \\ ${ }^{1}$ Department of Mathematical Sciences, University of Cincinnati, PO Box 210025, Cincinnati, OH 45221 - \\ 0025, USA.E-mail: barrerjd@mail.uc.edu \\ ${ }^{2}$ Centre de Mathématiques Appliquées, École Polytechnique, Route de Saclay 91128, Palaiseau Cedex, \\ France. E-mail: david.barrera@polytechnique.edu
}

In this paper, we study the asymptotic behavior of the normalized cadlag functions generated by the discrete Fourier transforms of a stationary centered square-integrable process, started at a point.

We prove that the quenched invariance principle holds for averaged frequencies under no assumption other than ergodicity, and that this result holds also for almost every fixed frequency under a certain generalization of the Hannan condition and a certain rotated form of the Maxwell and Woodroofe condition which, under a condition of weak dependence that we specify, is guaranteed for a.e. frequency. If the process is in particular weakly mixing, our results describe the asymptotic distributions of the normalized discrete Fourier transforms at every frequency other than 0 and $\pi$ under the generalized Hannan condition.

We prove also that under a certain regularity hypothesis the conditional centering is irrelevant for averaged frequencies, and that the same holds for a given fixed frequency under the rotated Maxwell and Woodroofe condition but not necessarily under the generalized Hannan condition. In particular, this implies that the hypothesis of regularity is not sufficient for functional convergence without random centering at a.e. fixed frequency.

The proofs are based on martingale approximations and combine results from Ergodic theory of recent and classical origin with approximation results by contemporary authors and with some facts from Harmonic Analysis and Functional Analysis.

Keywords: central limit theorem; discrete Fourier transform; invariance principle; martingale approximation; quenched convergence

\section{Introduction}

A recent result by Barrera and Peligrad ([5], Theorem 1) shows that the quenched central limit theorem holds for the normalized components of the Fourier transforms of a stationary process in $L^{2}$ orthogonal to the subspace of functions that are measurable with respect to the initial sigma algebra. This quenched limit theorem corresponds to previous, annealed limit theorems developed first by $\mathrm{Wu}$ in [23] and improved later by Peligrad and Wu in [20].

The paper [20] contains also an invariance principle ([20], Proposition 2.1) for the Fourier Transforms. In [5], on the other side, the problem of quenched convergence for the sample paths 
generated by the discrete Fourier transforms is not studied, which in particular leaves untouched the problem of extending to the quenched setting the aforementioned invariance principle by Peligrad and $\mathrm{Wu}$.

In this paper, we will address this problem. We will prove an "averaged frequency" quenched limit theorem (Theorem 2) corresponding to the invariance principle by Peligrad and Wu. We will also see that the asymptotic behavior of the normalized sample paths started at a point can be described for almost every fixed frequency under a certain "weak form" of the Hannan condition (see (37)) and under a certain "fixed frequency form" of the Maxwell and Woodroofe condition (see (39)).

More specifically, we will see that, under the weakened Hannan condition (37), martingale approximations are possible for every frequency other than (perhaps) zero, and we will deduce and describe asymptotic distributions for the normalized sample paths at every frequency other than those corresponding to the "square root" of the point spectrum of the Koopman operator associated to the process. We will also see that the same conclusion holds for a fixed frequency in this set provided that (39) holds, with the additional conclusion that, in this case, the random centering is not needed. We will give a sufficient condition, (42), for the fulfillment of (39) at a.e. frequency.

We emphasize that no assumption of regularity (see (29)) is needed for this, though some of the proofs are reached by first reducing to the regular case. We also emphasize that our proofs take advantage of some recently developed techniques in the realm of the calculus of asymptotic distributions. See, for instance, Lemma 5.6 and its proof, and the results by Cuny and Volný in [10]. We point out as well that the forthcoming results are valid for complex-valued processes: the estimates needed for the martingale approximations do not require the special properties (for instance, total ordering) of the real numbers in an essential way, ${ }^{1}$ and the martingale limit theorems are valid as far as the square root of the point spectrum is avoided for the rotations.

Our presentation is organized as follows: in Section 2, we will introduce some general facts and notions related to the quenched convergence of stochastic processes. Then we will introduce, in Section 3, the setting in which our discussions will take place. Section 4 presents briefly the essentials of convergence in distribution for complex-valued cadlag functions. In Section 5, we will present without proof our main results, Theorems 2, 3 and 4, preceded by a brief series of martingale approximation lemmas needed for their proofs. Section 6 is devoted to the martingale case, and Sections 7 and 8 are devoted, respectively, to the proofs of Theorems 2 and 3 together with 4. Finally, the Appendix presents some results that are used along the previous proofs and that deserve special mention due to their general or classical nature.

Notation. Throughout this paper, $\mathbb{N}=\{0,1,2, \ldots\}$ denotes the natural numbers starting at zero, we also use the notation $\mathbb{N}^{*}:=\mathbb{N} \backslash\{0\}$. Unless otherwise specified, an expression of the form " $\lim _{n}$ " (or " $\limsup _{n}$ " or " $\liminf _{n}$ ") and " $\rightarrow_{n}$ " must be read as " $\lim _{n \rightarrow \infty}$ " (and similarly for "lim $\sup _{n}$ " and "liminf $n$ ") and " $\rightarrow n \rightarrow \infty "$ ".

\footnotetext{
${ }^{1}$ The same observation applies to many of the results referenced in this paper, which are often stated under the unnecessary assumption that the processes under consideration are real-valued.
} 


\section{Quenched convergence}

Let $\left(Y_{n}\right)_{n}$ be a measurable sequence on some metric space $(S, d)$. This is, for every $n(n \in \mathbb{N}$ or $\mathbb{N}^{*}$ or $\left.\mathbb{Z}\right), Y_{n}:(\Omega, \mathcal{F}) \rightarrow(S, \mathcal{S})$ is an $\mathcal{F} / \mathcal{S}$-measurable function where $(\Omega, \mathcal{F})$ is a (fixed) measurable space and $\mathcal{S}$ is the Borel sigma algebra of $S$. Let $\mathbb{P}$ be a given probability measure on $(\Omega, \mathcal{F})$, so that $(\Omega, \mathcal{F}, \mathbb{P})$ is a probability space, and denote by " $\Rightarrow \mathbb{P}$ " the convergence in distribution with respect to $\mathbb{P}$.

The Portmanteau theorem ([7], Theorem 2.2) states, among other equivalences, that if $Y$ : $\left(\Omega^{\prime}, \mathcal{F}^{\prime}, \mathbb{P}^{\prime}\right) \rightarrow(S, \mathcal{S})$ is a random element of $S$, then $Y_{n} \Rightarrow_{\mathbb{P}} Y$ (as $\left.n \rightarrow \infty\right)$ if and only if for every continuous and bounded function $f: S \rightarrow \mathbb{R}$

$$
\int_{\Omega} f \circ Y_{n}(\omega) d \mathbb{P}(\omega) \rightarrow_{n} \int_{\Omega^{\prime}} f \circ Y(z) d \mathbb{P}^{\prime}(z),
$$

or, in the usual probabilistic notation, if and only if

$$
\lim _{n} E f\left(Y_{n}\right)=E f(Y)
$$

where $E$ is the expectation (Lebesgue integral) with respect to the corresponding probability measures $^{2}$ and $f(Z):=f \circ Z$ (whenever this makes sense).

A stronger kind of convergence, quenched convergence, can be defined in the following way: fix a sub-sigma algebra $\mathcal{F}_{0} \subset \mathcal{F}$ representing, in a heuristic language, the "initial information" about (or the "initial conditions of") the process $\left(Y_{n}\right)_{n}$, and denote by $E_{0}$ the conditional expectation with respect to $\mathcal{F}_{0}$. Then we will say that $Y_{n}$ converges to $Y$ in the quenched sense with respect to $\mathcal{F}_{0}$ if for every continuous and bounded function $f: S \rightarrow \mathbb{R}$

$$
E_{0}\left[f\left(Y_{n}\right)\right] \rightarrow_{n} E f(Y), \quad \mathbb{P} \text {-a.s. }
$$

Note that since this is pointwise convergence of uniformly bounded functions (to a constant value), the dominated convergence theorem guarantees that $\lim _{n} E f\left(Y_{n}\right)=E f(Y)$, thus quenched convergence implies convergence in distribution.

Remark 2.1. The same argument, in combination with Theorem 34.2(v) in [6], shows that if $\mathcal{G}_{0} \subset \mathcal{F}_{0}$ is any sigma algebra, then the assumption of quenched convergence with respect to $\mathcal{F}_{0}$ implies that $\lim _{n} E\left[f\left(Y_{n}\right) \mid \mathcal{G}_{0}\right]=E f(Y), \mathbb{P}$-a.s. In other words, quenched convergence with respect to a given sigma algebra $\mathcal{F}_{0}$ implies quenched convergence with respect to any subsigma algebra of $\mathcal{F}_{0}$. Note also that one can interpret convergence in distribution (or "annealed" convergence) as quenched convergence with respect to the trivial sigma algebra $\{\varnothing, \Omega\}$.

An example showing that the notion of quenched convergence is strictly stronger than convergence in distribution can be constructed by starting from any sequence $\left(Y_{n}\right)_{n}$ of $\mathcal{F}_{0}$-measurable functions and noticing that quenched convergence of $Y_{n}$ to $Y$ in this case is the same as

\footnotetext{
${ }^{2}$ When necessary, we will indicate the underlying measures in some specified way, writing for instance, " $E_{\mathbb{P}}$ " instead of " $E$ " for the expectation with respect to $\mathbb{P}$.
} 
$f\left(Y_{n}\right) \rightarrow E f(Y), \mathbb{P}$-a.s., for all continuous and bounded functions $f$, which is not possible if, for instance, $\left(Y_{n}\right)_{n}$ takes the values 1 and 0 infinitely often, $\mathbb{P}$-a.s. ${ }^{3}$

More specifically, consider a sequence $\left(Y_{n}\right)_{n}$ that converges in distribution but gives $\mathbb{P}$-a.s. a sequence with infinitely many 0 s and $1 \mathrm{~s}$, and define $\mathcal{F}_{0}:=\sigma\left(\left\{Y_{n}\right\}_{n}\right)$. For instance, take the unit interval $[0,1]$ with the Lebesgue measure on its Borel sigma algebra as the underlying probability space and let $Y_{n}:[0,1] \rightarrow\{0,1\}$ be the characteristic function of $[0,1 / 2)$ or the characteristic function of $[1 / 2,1]$ according to whether $n$ is, respectively, even or odd. For another example, closely related to the content of this paper, the reader is referred to [3].

Now assume that $E_{0}$ is a regular conditional expectation: there exists a family of probability measures $\left\{\mathbb{P}_{\omega}\right\}_{\omega \in \Omega}$ such that for every integrable $X:(\Omega, \mathcal{F}, \mathbb{P}) \rightarrow \mathbb{R}$

$$
\omega \mapsto \int_{\Omega} X(z) d \mathbb{P}_{\omega}(z)
$$

defines an $\mathcal{F}_{0}$-measurable version of $E_{0} X{ }^{4}$ The existence of such a family is guaranteed if, for instance, $(\Omega, \mathcal{F})$ is a Borel space, regardless of what $\mathcal{F}_{0}$ is. See Theorem 5.14 in [15].

From now on, we will just say that $Y_{n}$ converges to $Y$ in the quenched sense to mean that the quenched convergence is with respect to a fixed sigma algebra $\mathcal{F}_{0}$, returning to the full description only if necessary to avoid ambiguity. Our first result on quenched convergence is the following:

Proposition 2.2. Assume that $S$ is separable. If $E_{0}$ is regular and $Y_{n}$ converges to $Y$ in the quenched sense ( $Y$ is defined on some probability space $\left(\Omega^{\prime}, \mathcal{F}^{\prime}, \mathbb{P}^{\prime}\right)$ ), there exists a set $\Omega_{0} \subset \Omega$ with $\mathbb{P} \Omega_{0}=1$ such that for all $f: S \rightarrow \mathbb{R}$ continuous and bounded and all $\omega \in \Omega_{0}$

$$
\int_{\Omega} f \circ Y_{n}(z) d \mathbb{P}_{\omega}(z) \rightarrow_{n} \int_{\Omega^{\prime}} f \circ Y(z) d \mathbb{P}^{\prime}(z) .
$$

In particular, $Y_{n}$ converges to $Y$ in the quenched sense if and only if for $\mathbb{P}$-a.e. $\omega, Y_{n} \Rightarrow \mathbb{P}_{\omega} Y$ as $n \rightarrow \infty$.

Thus, we can choose the set of a.s. convergence in the definition of quenched convergence uniform over $\mathbf{C}^{b}(S)$ : the space of bounded, continuous functions $S \rightarrow \mathbb{R}$. The set $\Omega_{0}$ depends, nonetheless, on $\left(Y_{n}\right)_{n}$.

${ }^{3}$ Notice that, in this case $\left(f\left(Y_{n}\right)\right)_{n}$ has no limit whatsoever, $\mathbb{P}$-a.s., for any $f$ with $f(0) \neq f(1)$.

${ }^{4}$ More precisely we require, for a fixed version of $X$, the existence of an $\mathcal{F}_{0}$-measurable set $\Omega_{X}$ with $\mathbb{P} \Omega_{X}=1$ such that (4) makes sense for every $\omega \in \Omega_{X}$, and such that the function given by (4) if $\omega \in \Omega_{X}$, and zero otherwise, defines an $\mathcal{F}_{0}$-measurable version of $E_{0} X$, i.e., an $\mathcal{F}_{0}$-measurable function $\tilde{X}$ satisfying

$$
\int_{A} \tilde{X}(\omega) d \mathbb{P}(\omega)=\int_{A} X(\omega) d \mathbb{P}(\omega)
$$

for every $A \in \mathcal{F}_{0}$. It is possible to prove the existence of such $\Omega_{X}$ just by requiring that the function defined by (4) if $X \in L_{\mathbb{P}_{\omega}}^{1}$ and by zero otherwise defines an $\mathcal{F}_{0}$-measurable version of $E_{0} X$ for every $X \in L_{\mathbb{P}}^{1}$. See, for instance, [4], Remark 11.2. The approximation argument for this statement, which is similar to the one used to prove Lemma A.11 in the Appendix, actually shows that it is enough to require that for every $A \in \mathcal{F}, \omega \mapsto \mathbb{P}_{\omega}(A)$ defines an $\mathcal{F}_{0}$-measurable version of $\mathbb{P}\left(A \mid \mathcal{F}_{0}\right)$. 
Proof of Proposition 2.2. Denote by $E^{\omega}$ the integration with respect to $\mathbb{P}_{\omega}$, and consider functions $U_{k, \epsilon}$ as in the statement 2. of Lemma A.1 in the Appendix. By the definition of regularity there exists, for all $k \in \mathbb{N}$ and all $\in \in \mathbb{Q} \cap(0, \infty)$, a set $\Omega_{k, \epsilon} \subset \Omega$ with $\mathbb{P} \Omega_{k, \epsilon}=1$ such that

$$
\Omega_{k, \epsilon} \subset\left\{\omega \in \Omega: E^{\omega} U_{k, \epsilon}\left(Y_{n}\right) \rightarrow_{n} E U_{k, \epsilon}(Y)\right\} .
$$

Now take $\Omega_{0}:=\bigcap_{k, \epsilon} \Omega_{k, \epsilon}$ and use Lemma A.1.

The importance of Proposition 2.2 resides, for us, in the following fact: to prove results on quenched convergence we will apply some classical theorems to the processes involved in our arguments seen as stochastic processes under the measures $\mathbb{P}_{\omega}$. Without this result the uniformity of $\Omega_{0}$, which is eventually necessary, would require a case-by-case approach, making the proofs much longer and less transparent.

Remark 2.3. We also point out the following: a set has $A \in \mathcal{F}$ satisfies $\mathbb{P} A=1$ if and only if $\mathbb{P}_{\omega} A=1$ for $\mathbb{P}$-a.e. $\omega$. To see this, use the equality

$$
\mathbb{P} A=\int_{\Omega} \mathbb{P}_{\omega} A d \mathbb{P}(\omega) .
$$

\section{General setting}

\subsection{Assumptions}

Our general setting, fixed from now on along this paper, will be the following: first, denote by $\lambda$ the normalized Lebesgue measure in the Borel sigma algebra $\mathcal{B}$ of $[0,2 \pi)$. This is,

$$
\lambda(A)=\frac{1}{2 \pi} L(A)
$$

for every $A \in \mathcal{B}$, where $L$ is the Lebesgue measure on $\mathcal{B}$.

By a random variable we mean a complex-valued measurable function $Y: \Omega \rightarrow \mathbb{C}$ defined on some probability space $(\Omega, \mathcal{F}, \mathbb{P})$.

Next, let $\left(X_{k}\right)_{k \in \mathbb{Z}}$ be a strictly stationary, ergodic sequence of random variables defined on a probability space $(\Omega, \mathcal{F}, \mathbb{P})$. This is: $X_{k}=X_{0} \circ T^{k}$, where $T: \Omega \rightarrow \Omega$ is an ergodic, invertible, and bimeasurable transformation.

We will assume that $X_{0} \in L_{\mathbb{P}}^{2}\left(\mathcal{F}_{0}\right)$ where $\mathcal{F}_{0} \subset \mathcal{F}$ is a sigma algebra satisfying $\mathcal{F}_{0} \subset T^{-1} \mathcal{F}_{0}$ (i.e. $T^{-1}$ is $\mathcal{F}_{0}$-measurable), and we define $\mathcal{F}_{n}:=T^{-n} \mathcal{F}_{0}$ for all $n \in \mathbb{Z}, \mathcal{F}_{-\infty}:=\bigcap_{n \in \mathbb{Z}} \mathcal{F}_{n}$, and $\mathcal{F}_{\infty}:=\sigma\left(\bigcup_{n \in \mathbb{Z}} \mathcal{F}_{n}\right)$. Thus $\left(\mathcal{F}_{k}\right)_{k=-\infty}^{\infty}$ is an increasing $T$-filtration: $\mathcal{F}_{k} \subset \mathcal{F}_{k+1}$ and $T^{-l} \mathcal{F}_{k}=$ $\mathcal{F}_{k+l}$.

For any $n \in\{-\infty\} \cup \mathbb{Z}$, denote by $E_{n}$ the conditional expectation with respect to $\mathcal{F}_{n}$, thus $E_{n} Z:=E\left[Z \mid \mathcal{F}_{n}\right]$ for every $\mathbb{P}$-integrable $Z$, and let the projection $\mathcal{P}_{n}$ be given by

$$
\mathcal{P}_{n} Y:=E_{n} Y-E_{n-1} Y \text {. }
$$


Note that for $Y \in L_{\mathbb{P}}^{2}, \mathcal{P}_{n} Y \in L_{\mathbb{P}}^{2}\left(\mathcal{F}_{n}\right) \ominus L_{\mathbb{P}}^{2}\left(\mathcal{F}_{n-1}\right)$. This is, $\mathcal{P}_{n} Y$ is $\mathcal{F}_{n}$-measurable and $E_{n-1} \mathcal{P}_{n} Y=0$.

Assume also that $E_{0}$ is a regular conditional expectation: as explained in Section 2, there exists a family of probability measures $\left\{\mathbb{P}_{\omega}\right\}_{\omega \in \Omega}$ such that for every integrable function $X$,

$$
\omega \mapsto \int_{\Omega} X(z) d \mathbb{P}_{\omega}(z)
$$

defines an $\mathcal{F}_{0}$-measurable version of $E_{0} X$ (see also the footnote following (1310)).

We will finally assume that $\mathcal{F}_{0}$ and $\mathcal{F}$ are countably generated or, alternatively, that $\mathcal{F}_{0}$ is countably generated and $\mathcal{F}=\mathcal{F}_{\infty}$ (so $\mathcal{F}$ is also countably generated). This alternative is possible because we will deal only with $\mathcal{F}_{\infty}$-measurable functions.

Remark 3.1. If we also denote by $T: L_{\mathbb{P}}^{1} \rightarrow L_{\mathbb{P}}^{1}$ the Koopman operator associated to $T$, namely $T Y:=Y \circ T$ then, clearly, $X_{n}=T^{n} X_{0}$ for all $n$, and it is not hard to see, using stationarity, that

$$
T^{r} E_{s}=E_{s+r} T^{r}
$$

(as operators in $L_{\mathbb{P}}^{1}$ ) for all integers $r, s$. Similarly, an application of the reverse martingale convergence theorem (see for instance Theorem 5.6.1 and Exercise 5.6.1 in [14]) shows that, for every $n \in \mathbb{Z}$,

$$
T^{n} E_{-\infty}=E_{-\infty} T^{n}
$$

It is important to point out also that, again by the reverse martingale convergence theorem, the following holds: for every $X \in L_{\mathbb{P}}^{p}(p \geq 1)$

$$
E_{-\infty} X=\lim _{n} E_{-n} X
$$

$\mathbb{P}$-a.s. and in $L_{\mathbb{P}}^{p}$.

Remark 3.2. We also recall the following fact about the Koopman operator $T$ : under ergodicity, the eigenvalues of $T$ form a subgroup of $\mathbb{T}$, the unit circle seen as a (Lie) group under the operation of multiplication of complex numbers (see [16], Proposition 7.17). We will denote this group by $\operatorname{Spec}_{p}(T)$, the point spectrum of $T$. Note that since $L_{\mathbb{P}}^{2}$ admits a countable orthonormal basis ( $\mathcal{F}$ is countably generated) and the eigenspaces of $T$ are mutually orthogonal ( $T$ is measure preserving), $\operatorname{Spec}_{p}(T)$ is countable. In particular,

$$
\lambda\left(\left\{\theta \in[0,2 \pi): e^{i \theta} \in \operatorname{Spec}_{p}(T)\right\}\right)=0 .
$$

\subsection{Quenched convergence in the product space}

There is a special form of quenched convergence that will be of interest to us: let $\mathcal{G}_{0}:=\mathcal{B}_{0} \otimes \mathcal{F}_{0} \subset$ $\mathcal{B} \otimes \mathcal{F}$ where $\mathcal{B}_{0} \subset \mathcal{B}$ is a given sigma algebra (we will choose $\mathcal{B}_{0}=\mathcal{B}$ or $\mathcal{B}_{0}=\{\varnothing,[0,2 \pi)\}$ according to the problem under consideration). 
Assuming that $E\left[\cdot \mid \mathcal{B}_{0}\right]$ (conditional expectation with respect to $\lambda$ ) is regular with regular measures $\left\{\lambda_{\theta}\right\}_{\theta \in[0,2 \pi)}$, an application of Corollary A.12 in the Appendix shows that, for any $\mathcal{B} \otimes \mathcal{F}$ integrable function $Y=Y(\theta, \omega)$, a version of $E\left[Y \mid \mathcal{G}_{0}\right]$ is given by

$$
E\left[Y \mid \mathcal{G}_{0}\right](\theta, \omega)=\int_{[0,2 \pi)} E^{\omega}[Y(x, \cdot)] d \lambda_{\theta}(x),
$$

where $\omega \mapsto E^{\omega} Y(x, \cdot)$ is the version of $E_{0}[Y(x, \cdot)]$ given by (7). From now on, we will denote by $\tilde{E}_{0}$ the conditional expectation $E\left[\cdot \mid \mathcal{G}_{0}\right]$ (again, we will carry on discussions under the two choices $\mathcal{B}_{0}=\{\varnothing,[0,2 \pi)\}$ and $\left.\mathcal{B}_{0}=\mathcal{B}\right)$. Whenever needed, we will work under the regular version of $\tilde{E}_{0}$, so that for any integrable $Y=Y(\theta, \omega)$

$$
\tilde{E}_{0}[Y](\theta, \omega)=\int_{[0,2 \pi)} \int_{\Omega} Y(x, z) d \mathbb{P}_{\omega}(z) d \lambda_{\theta}(x) .
$$

Remark 3.3. Note that if $\mathcal{B}_{0}=\{\varnothing,[0,2 \pi)\}$ is the trivial sigma algebra, then $\lambda_{\theta}:=\lambda$ for all $\theta \in[0,2 \pi)$ defines a family of regular measures for $E\left[\cdot \mid \mathcal{B}_{0}\right]$. And that in this case $\tilde{E}_{0} Y$, being constant in $\theta$ (for a fixed $\omega$ ), defines an $\mathcal{F}_{0}$-measurable function. In particular, $\tilde{E}_{0} Y$ can be regarded, in this case, as a random variable defined on $(\Omega, \mathcal{F}, \mathbb{P})$.

\section{The space $D[[0, \infty), \mathbb{C}]$}

This paper deals with convergence in distribution, under several measures, of random elements of $D[[0, \infty), \mathbb{C}]$ : the space of functions $f:[0, \infty) \rightarrow \mathbb{C}$ that are continuous from the rightand have left-hand limits at every point (complex-valued cadlag functions). This space is an algebra with the operation of multiplication and addition given by the usual pointwise operations between complex-valued functions, and it is a $(\mathbb{C}$ or $\mathbb{R}$-)vector space with the usual operation of multiplication by constants regarded as constant functions.

Let $D[0, \infty)$ denote the space of real-valued cadlag functions $f:[0, \infty) \rightarrow \mathbb{R}$ (as presented for instance in [7], Section 16). If we denote by $(\operatorname{Re}(f), \operatorname{Im}(f))$ the vector of real and imaginary parts of a function $f \in D[[0, \infty), \mathbb{C}]$, the bijection $D[[0, \infty), \mathbb{C}] \rightarrow D[0, \infty) \times D[0, \infty)$ given by

$$
f \mapsto(\operatorname{Re}(f), \operatorname{Im}(f))
$$

allows us to regard $D[[0, \infty), \mathbb{C}]$ as a topological space whose topology is the topology generated by the product Skorohod topology of $D[0, \infty) .{ }^{5}$ In particular $D[[0, \infty), \mathbb{C}]$ is separable and metrizable. If we use the product metric

$$
d(f, g):=\left((d(\operatorname{Re}(f), \operatorname{Re}(g)))^{2}+(d(\operatorname{Im}(f), \operatorname{Im}(g)))^{2}\right)^{1 / 2},
$$

where $d$ (at the right) is the Skorohod distance defined by [7], $(12.16)$ then $D[[0, \infty), \mathbb{C}]$ is complete. A similar construction shows the corresponding facts for the space $D[[0, m], \mathbb{C}]$ of functions that are restrictions of elements in $D[[0, \infty), \mathbb{C}]$ to the interval $[0, m]$.

\footnotetext{
${ }^{5}$ But remember that this is not a topological vector space. See exercise 12.2 in [7].
} 
Denote by $\mathcal{D}_{\infty, \mathbb{C}}$ the Borel sigma algebra of $D[[0, \infty), \mathbb{C}]$. The following observations will suffice to clarify the proofs of convergence in distribution given here:

1. First, if $Y:(\Omega, \mathcal{F}, \mathbb{P}) \rightarrow D[[0, \infty), \mathbb{C}]$ is a random function (this is, an $\mathcal{F} / \mathcal{D}_{\infty, \mathbb{C}}$ measurable function, where $(\Omega, \mathcal{F}, \mathbb{P})$ is a probability space), then the inequalities

$$
\mathbb{P}\left[Y_{n} \notin K_{1} \times K_{2}\right] \leq \mathbb{P}\left[\operatorname{Re}\left(Y_{n}\right) \notin K_{1}\right]+\mathbb{P}\left[\operatorname{Im}\left(Y_{n}\right) \notin K_{2}\right] \leq 2 \mathbb{P}\left[Y_{n} \notin K_{1} \times K_{2}\right]
$$

show that a sequence of random elements $\left(Y_{n}\right)_{n}$ in $D[[0, \infty), \mathbb{C}]$ is tight if and only if $\left(\operatorname{Re}\left(Y_{n}\right)\right)_{n}$ and $\left(\operatorname{Im}\left(Y_{n}\right)\right)_{n}$ are tight.

2. By an adaptation of the arguments in [7], it is possible to show that the finite dimensional distributions are a separating class in $D[[0, \infty), \mathbb{C}]$ : if for every $t$ we denote by $\pi_{t}$ the coordinate function $\pi_{t}(x):=x(t)$, then two probability measures $\mathbb{P}_{1}$ and $\mathbb{P}_{2}$ in $D[[0, \infty), \mathbb{C}]$ coincide if and only if there exists a dense subset $J \subset[0, \infty)$ such that for every $0 \leq t_{1} \leq \cdots \leq t_{n}$ in $J$ the $n$th dimensional distributions $\left(\mathbb{P}_{j} \pi_{t_{1}}^{-1}, \ldots, \mathbb{P}_{j} \pi_{t_{n}}^{-1}\right)(j=1,2)$ on $\mathbb{C}^{n}=\mathbb{R}^{2 n}$ are the same. In particular, one can prove that $\mathbb{P}_{n} \Rightarrow_{n} \mathbb{P}$ (" $\Rightarrow$ " denotes weak convergence of measures) by proving tightness and convergence of the finite-dimensional distributions, and if $m>0$ is such that

$$
\mathbb{P}\left\{x: \lim _{t \rightarrow m^{-}} x(t) \neq x(m)\right\}=0
$$

and $r_{m}: D[[0, \infty), \mathbb{C}] \rightarrow D[[0, m], \mathbb{C}]$ is the restriction operator ${ }^{6}\left(r_{m} x(t)=x(t)\right)$, then $\mathbb{P}_{n} \Rightarrow \mathbb{P}$ in $D[[0, \infty), \mathbb{C}]$ implies that $\mathbb{P}_{n} r_{m}^{-1} \Rightarrow \mathbb{P r}_{m}^{-1}$ in $D[[0, m], \mathbb{C}]$. Conversely, if $\left(m_{k}\right)_{k}$ is a sequence increasing to infinity such that (13) holds for all $m=m_{k}$, then $\mathbb{P}_{n} r_{m_{k}}^{-1} \Rightarrow \operatorname{Pr}_{m_{k}}^{-1}$ (on $D\left[\left[0, m_{k}\right], \mathbb{C}\right]$ ) for all $k$ implies that $\mathbb{P}_{n} \Rightarrow_{n} \mathbb{P}$.

Let us finally mention that, by our definition of the topology of $D[[0, \infty), \mathbb{C}]$, to prove that a function $Y: \Omega \rightarrow D[[0, \infty), \mathbb{C}]$ defined on a measurable space $(\Omega, \mathcal{F})$ is $\mathcal{F} / \mathcal{D}_{\infty, \mathbb{C}}$ measurable it suffices to see the measurability of the real and imaginary parts of $Y$. This observation, combined with the argument in [7], p. 84, and with Theorem 16.6 in that book shows that $Y$ is $\mathcal{F} / \mathcal{D}_{\infty, \mathbb{C}^{-}}$ measurable if for every $t \in[0, \infty), \omega \mapsto Y(\omega)(t)$ is $\mathcal{F}$-measurable.

\section{Results and comments}

In this section, we will present the main results of this paper. Some of the proofs are not difficult and can be given after the statements. More technically demanding facts will be deferred to later sections.

Let us start by introducing the notion of discrete Fourier transforms.

Definition 5.1. Let $T: \Omega \rightarrow \Omega$ be an invertible, bimeasurable, measure-preserving transformation on a probability space $(\Omega, \mathcal{F}, \mathbb{P})$, let $Y_{0}: \Omega \rightarrow \mathbb{C}$ be a random variable, and let $Y_{k}:=T^{k} Y_{0}$

\footnotetext{
${ }^{6}$ The space $D[[0, m], \mathbb{C}]$ is defined via the product metric as above starting from the space $D[0, m]$ of real-valued cadlag functions $f:[0, m] \rightarrow \mathbb{R}$. See (again) Section 16 in [7] or Section 7 in [4] for more details on the last space.
} 
$\left(k \in \mathbb{N}\right.$ or $\mathbb{Z}$ ). For every $\theta \in[0,2 \pi)$ and $n \in \mathbb{N}^{*}$, the $n$th discrete Fourier Transform of $\left(Y_{k}\right)_{k}$ at the frequency $\theta$ is defined by

$$
S_{n}\left(Y_{0}, T, \theta, \omega\right):=\sum_{k=0}^{n-1} e^{i k \theta} Y_{k}(\omega)
$$

When $Y_{0}$ and $T$ are fixed and $\theta \in(0,2 \pi)$ is given, we will denote by $S_{n}(\theta)$ (or $\left.S_{n}(\theta, \cdot)\right)$ the random variable $S_{n}\left(Y_{0}, T, \theta, \cdot\right)$. If in addition $\theta=0$, we denote by $S_{n}$ the random variable $S_{n}\left(Y_{0}, T, 0, \cdot\right)$. So $S_{n}(\omega):=\sum_{k=0}^{n-1} Y_{k}(\omega)$.

Remark 5.2. We strengthen the fact that, at this point, $(\Omega, \mathcal{F}, \mathbb{P})$ and $T$ in this definition are not necessarily the objects fixed in Section 3.1, though we will not go "too far away" from them, ${ }^{7}$ hence the specification of $T$ in the notation.

Nonetheless, throughout the rest of this section, we will work under the assumptions specified in Section 3.1. In particular, we will not specify $T$ in the notation for discrete Fourier transforms.

We will address the problem of quenched convergence for the cadlag random functions generated by the discrete Fourier transforms of $\left(X_{k}\right)_{k}$. Let us start by recalling the following theorem, proved in [5]:

Theorem 1. There exists a set $I \subset(0,2 \pi)$ with $\lambda(I)=1$ such that, for all $\theta \in I$, the random variables

$$
\frac{1}{\sqrt{n}}\left(S_{n}(\theta)-E_{0} S_{n}(\theta)\right)
$$

converge in the quenched sense, as $n \rightarrow \infty$, to a complex Gaussian random variable with independent real and imaginary parts, each with mean zero and variance $\sigma^{2}(\theta) / 2$, where

$$
\sigma^{2}(\theta)=\lim _{n} \frac{E_{0}\left|S_{n}(\theta)-E_{0} S_{n}(\theta)\right|^{2}}{n}=\lim _{n} \frac{E\left|S_{n}(\theta)-E_{0} S_{n}(\theta)\right|^{2}}{n},
$$

$\mathbb{P}$-a.s. (thus the limit exists with probability one, and it is nonrandom).

We will refer to this as the quenched central limit theorem for the discrete Fourier transforms of a stationary process. Our main goal in this paper is to explore possible extensions of this theorem to corresponding quenched invariance principles.

Remark 5.3. The function $\theta \mapsto \sigma^{2}(\theta)$ defines a version of the spectral density, with respect to $\lambda$, of the process $\left(X_{k}-E_{-\infty} X_{k}\right)_{k}$. For details on this, see the proof of Theorem 15.1 in [4] (or combine Remark 5.8 and (84) below with the proof of Theorem 3 in [19]).

\footnotetext{
${ }^{7}$ Indeed, we will need to work with product spaces and product maps along some of the proofs and this makes convenient to relax these objects in Definition 5.1.
} 
In order to discuss the issue of quenched functional convergence, let us define the following random variables, which will appear again later along the proofs:

$$
D_{r, 0}(\theta):=\sum_{k=0}^{r} \mathcal{P}_{0} X_{k} e^{i k \theta},
$$

and

$$
M_{r, n}(\theta):=\sum_{k=0}^{n-1} T^{k} D_{r, 0}(\theta) e^{i k \theta} .
$$

Where $0 \leq r<\infty$ and we allow the value $r=\infty$ when $D_{\infty, 0}(\theta)$ makes sense as the limit $(r \rightarrow \infty) \mathbb{P}$-a.s. and in $L_{\mathbb{P}}^{2}$ of $D_{r, 0}(\theta)$. Denote also by $V_{r, n}(\theta)$ the random function

$$
V_{r, n}(\theta)(t):=\frac{1}{\sqrt{n}} M_{r,\lfloor n t\rfloor}(\theta),
$$

and by $W_{n}(\theta)$ the random function

$$
W_{n}(\theta)(t):=\frac{S_{\lfloor n t\rfloor}(\theta)-E_{0} S_{\lfloor n t\rfloor}(\theta)}{\sqrt{n}} .
$$

We will also consider the non-centered version of $W_{n}(\theta)$

$$
U_{n}(\theta)(t):=W_{n}(\theta)(t)+\frac{E_{0} S_{\lfloor n t\rfloor}(\theta)}{\sqrt{n}}=\frac{S_{\lfloor n t\rfloor}(\theta)}{\sqrt{n}} .
$$

Remark 5.4. When necessary, especially when discussing quenched convergence in the product space $([0,2 \pi) \times \Omega, \mathcal{B} \otimes \mathcal{F})$, we will specify the dependence on $\theta \in[0,2 \pi)$ and $\omega \in \Omega$ by seeing these random elements as processes with two parameters (see Theorem 2 for example). So for instance,

$$
D_{r, 0}(\theta, \omega)=\sum_{k=0}^{r} \mathcal{P}_{0} X_{k}(\omega) e^{i k \theta}
$$

and so on.

\subsection{Approximation lemmas}

Recall the definition of the set $I$ specified in Theorem 1, see [5]; p. 289, 290: $\theta \in I$ if and only if

1. $e^{-2 i \theta} \notin \operatorname{Spec}_{p}(T)$ (equivalently, $e^{2 i \theta} \notin \operatorname{Spec}_{p}(T)$ ),

2. $E\left[\sup _{r}\left|\mathcal{P}_{0} S_{r}(\theta)\right|^{2}\right]<\infty$, and

3. $D_{\infty, 0}(\theta):=\lim _{n} \mathcal{P}_{0} S_{n}(\theta)$ exists $\mathbb{P}$-a.s. 
Notice that, for $\theta \in I, D_{\infty, 0}(\theta)$ is (also) the limit in $L_{\mathbb{P}}^{2}$ of $D_{r, 0}(\theta)$ : first, $D_{\infty, 0}(\theta) \in L_{\mathbb{P}}^{2}$ by the dominated convergence theorem and 2., and since $D_{r, 0}(\theta)-D_{\infty, 0}(\theta) \rightarrow 0, \mathbb{P}$-a.s. and $\mid D_{r, 0}(\theta)-$ $\left.D_{\infty, 0}(\theta)\right|^{2} \leq 2\left(\sup _{r}\left|D_{r, 0}(\theta)\right|^{2}+\left|D_{\infty, 0}(\theta)\right|^{2}\right)$ the dominated convergence theorem again implies that $D_{r, 0}(\theta) \rightarrow_{r} D_{\infty, 0}(\theta)$ in $L_{\mathbb{P}}^{2}$.

Lemma 5.5. Let $\operatorname{Re}(B), \operatorname{Im}(B): \Omega^{\prime} \rightarrow D[[0, \infty)]$ be independent standard Brownian motions 8 defined on some probability space $\left(\Omega^{\prime}, \mathcal{F}^{\prime}, \mathbb{P}^{\prime}\right)$, and consider the set of frequencies $\theta$ specified by condition 1 . above. Then for all such $\theta$ and all $0 \leq r<\infty, V_{r, n}(\theta)$ given by (19) converges in the quenched sense, as $n \rightarrow \infty$, to

$$
B_{r}(\theta):=\left(E\left|D_{r, 0}(\theta)\right|^{2} / 2\right)^{1 / 2}(\operatorname{Re}(B)+i \operatorname{Im}(B)) .
$$

If $\theta \in I$ ( $I$ is the set specified by 1.-3. above), then $V_{\infty, n}(\theta)$ converges in the quenched sense to

$$
B(\theta):=\left(E\left|D_{\infty, 0}(\theta)\right|^{2} / 2\right)^{1 / 2}(\operatorname{Re}(B)+i \operatorname{Im}(B)) .
$$

Proof. This is an immediate consequence of Theorem 5 in Section 6.

Our results will make use as well of the following "hypothetical" quenched invariance principle:

Lemma 5.6. If for a given $\theta \in[0,2 \pi)$, (19) converges in the quenched sense (with respect to $\mathcal{F}_{0}$ ) as $n \rightarrow \infty$ to a random function $B_{r}(\theta)$ for all but finitely many $r$ 's and

$$
\lim _{r} \limsup _{n} E_{0}\left[\frac{1}{n} \max _{1 \leq k \leq n}\left|S_{k}(\theta)-E_{0} S_{k}(\theta)-M_{r, k}(\theta)\right|^{2}\right]=0
$$

$\mathbb{P}$-a.s., then there exists a random function $B(\theta)$ such that $B_{r}(\theta)$ converges in distribution to $B(\theta)$ as $r \rightarrow \infty$, and (20) converges to $B(\theta)$ as $n \rightarrow \infty$ in the quenched sense. by

If, in particular, $e^{2 i \theta} \notin \operatorname{Spc}_{p}(T)$, then there exists a nonnegative number $\sigma(\theta)$ characterized

$$
\sigma^{2}(\theta):=\lim _{r} E\left|D_{r, 0}(\theta)\right|^{2}
$$

and

$$
B(\theta)=\frac{\sigma(\theta)}{\sqrt{2}}(\operatorname{Re}(B)+i \operatorname{Im}(B))
$$

(in distribution).

Note that if one agrees to call "standard" a $d$-dimensional Brownian motion $B=\left(B_{1}, \ldots, B_{d}\right)$ for which the entries are (independent and) identically distributed (centered) Brownian motions and such that $E|B(1)|^{2}=1$ where $|\cdot|$ is the Euclidean norm, the limit function (26) is just a standard 2-dimensional Brownian motion rescaled by $\sigma(\theta)$.

\footnotetext{
${ }^{8}$ The notation may be a little shocking. It is inspired in the fact that $B:=\operatorname{Re}(B)+i \operatorname{Im}(B)$ is a 2-dimensional Brownian motion.
} 
Remark 5.7. If we assume $a$ priori the existence of $B(\theta)$ with $B_{r}(\theta) \Rightarrow B(\theta)$ as $r \rightarrow \infty$, then the parts of the proof of this and analogous results that rely in Theorem A.2 in the Appendix can still be carried over using instead [7], Theorem 3.2.

This is possible for the values of $\theta$ that we will treat along the proofs of the results in this paper: we will be restricted to cases in which $D_{\infty, 0}(\theta)$ is well defined, but since our statement covers an (at least formally) more general case and the proofs via the result in [7] would be just the same (with the estimates used here), we proceed via Theorem A.2.

Remark 5.8. It is important to remark also the following: in the context of Lemma 5.6, (24) implies that, necessarily,

$$
\sigma^{2}(\theta)=\lim _{n} E_{0} \frac{1}{n}\left|S_{n}(\theta)-E_{0} S_{n}(\theta)\right|^{2}
$$

P-a.s.

Indeed, Lemma A.6 in the Appendix implies, by orthogonality, that

$$
E\left[\left|D_{r, 0}(\theta)\right|^{2}\right]=\lim _{n} E_{0} \frac{1}{n}\left|M_{r, n}(\theta)\right|^{2}
$$

$\mathbb{P}$-a.s. And (27) follows from (25) and (28) because, under (24)

$$
\begin{aligned}
& 0 \leq \limsup _{n}\left|\left(E_{0} \frac{1}{n}\left|S_{n}(\theta)-E_{0} S_{n}(\theta)\right|^{2}\right)^{1 / 2}-\sigma(\theta)\right|
\end{aligned}
$$

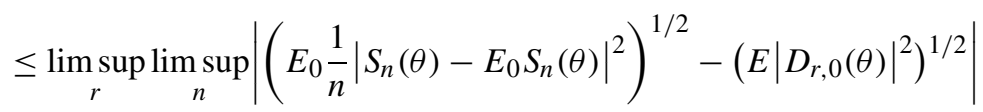

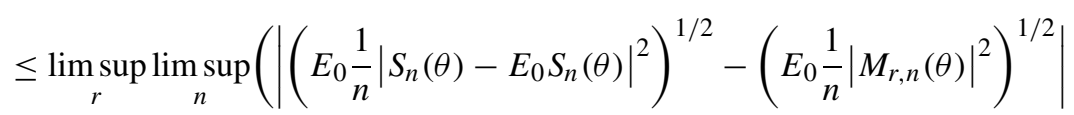

$$
\begin{aligned}
& \left.+\left|\left(E_{0} \frac{1}{n}\left|M_{r, n}(\theta)\right|^{2}\right)^{1 / 2}-\left(E\left|D_{r, 0}(\theta)\right|^{2}\right)^{1 / 2}\right|\right)
\end{aligned}
$$

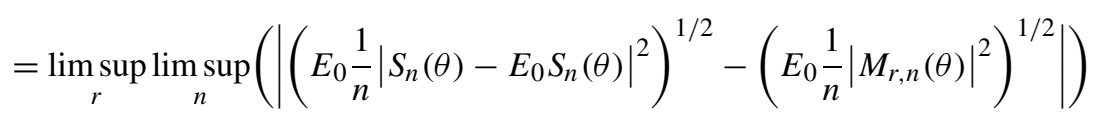

$$
\begin{aligned}
& \leq \limsup _{r} \limsup _{n}\left(E_{0} \frac{1}{n}\left|S_{n}(\theta)-E_{0} S_{n}(\theta)-M_{r, n}(\theta)\right|^{2}\right)^{1 / 2}=0,
\end{aligned}
$$

$\mathbb{P}$-a.s.

Proof of Lemma 5.6. For $m \geq 1$, the Skorohod metric $d_{m}$ on $D[[0, m], \mathbb{C}]$ is dominated by the uniform (product) metric. So

$$
d_{m}\left(r_{m} W_{n}(\theta), r_{m} V_{n}(\theta)\right) \leq \frac{\sqrt{m}}{\sqrt{n^{\prime}}} \max _{1 \leq k \leq n^{\prime}}\left|S_{k}(\theta)-E_{0} S_{k}(\theta)-M_{r, k}(\theta)\right|,
$$


where $n^{\prime}:=m n$ and " $r_{m}$ " is the restriction operator $D[[0, \infty), \mathbb{C}] \rightarrow D[[0, m], \mathbb{C}]$. It follows from (24) that

$$
\lim _{r} \limsup _{n}\left\|d_{m}\left(r_{m} W_{n}(\theta), r_{m} V_{r, n}(\theta)\right)\right\|_{\mathbb{P}_{\omega}, 2}=0
$$

for $\mathbb{P}$-almost every $\omega$. Thus by an application of Corollary A.3 in the Appendix, there exist random functions $B_{r}^{m}(\theta)$ and $B_{\infty}^{m}(\theta)$ in $D[[0, m], \mathbb{C}]$ such that the conclusion of Lemma 5.6 is satisfied with $B_{r}^{m}(\theta)$ in place of $B_{r}(\theta)$ and with $B_{\infty}^{m}(\theta)$ in place of $B(\theta)$. The first conclusion follows from a suitable adaptation of [7], p. 173, Lemma 3 (or likewise adapting the comments in 2. on Section 4).

To prove the second assertion, we argue as follows: first, for such $\theta$ 's, $B_{r}(\theta)$ is given by (22), and we have seen that the (quenched) limit of (20) as $n \rightarrow \infty$ is the same as that of $B_{r}(\theta)$ as $r \rightarrow \infty$.

Now, by convergence of Types theorems (see, for instance, [6], p. 193 or [3], Proposition 4), $B_{r}(\theta)$ admits a limit in distribution if and only if there exists

$$
\sigma^{2}(\theta):=\lim _{r} E\left|D_{r, 0}(\theta)\right|^{2},
$$

which leads us to conclude that (20) converges in the quenched sense to

$$
\frac{\sigma(\theta)}{\sqrt{2}}(\operatorname{Re}(B)+i \operatorname{Im}(B))
$$

as claimed.

Remark 5.9. Note that the convergence of (19) in the quenched sense as $n \rightarrow \infty$ is guaranteed for all $\theta \in I$ and all $0 \leq r \leq \infty$, where $I$ is the set specified by Theorem 1 . Whether the hypothesis (24) is true as well for $\lambda$-a.e. such $\theta$ is a question yet to be answered (it is without the " $\max _{1 \leq k \leq n}$ ", as can be seen from [5], p. 289, 290). Theorem 3 below gives particular cases of this statement.

It is important also to point out that $\left(X_{k}-E_{-\infty} X_{k}\right)$ is a regular process, according to the following definition:

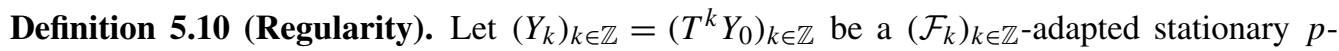
integrable process $(p \geq 1)$. The process $\left(Y_{k}\right)_{k \in \mathbb{Z}}$ is called regular if $E\left[Y_{0} \mid \mathcal{F}_{-k}\right]$ converges to 0 in $L_{\mathbb{P}}^{p}$. This is, if

$$
\lim _{k} E\left|E_{-k} Y_{0}\right|^{p}=\lim _{k} E\left|E_{0} Y_{k}\right|^{p}=0 .
$$

Equivalently, $\left(Y_{k}\right)_{k \in \mathbb{Z}}$ is regular if for every $k \in \mathbb{Z}$

$$
E\left[Y_{k} \mid \mathcal{F}_{-\infty}\right]=0
$$

Remark 5.11. As with the case of quenched convergence, the notion of regularity depends on the choice of the $T$-filtration $\left(\mathcal{F}_{k}\right)_{k \in \mathbb{Z}}$, but it is easy to show that if $\left(Y_{k}\right)_{k \in \mathbb{Z}}$ is regular with respect 
to some $T$-filtration $\left(\mathcal{F}_{k}^{\prime}\right)_{k \in \mathbb{Z}}$ with $\sigma\left(Y_{0}\right) \subset \mathcal{F}_{0}^{\prime}$, then it is regular with respect to its minimal $T$ filtration $\left(\mathcal{M}_{k}\right)_{k \in \mathbb{Z}}=\left(T^{-k} \mathcal{M}_{0}\right)_{k \in \mathbb{Z}}$, where

$$
\mathcal{M}_{0}=\bigcap_{\alpha} \mathcal{G}_{\alpha}
$$

with the intersection running over the sigma algebras $\mathcal{G}_{\alpha}$ in $\Omega$ for which $\sigma(Y) \subset \mathcal{G}_{\alpha} \subset T^{-1} \mathcal{G}_{\alpha}$. In this sense, the notion of regularity can be made independent of the choice of $\mathcal{F}_{0}$.

To prove the equivalence stated in Definition 5.10 note that, by (8), (9), (10) and the $L_{\mathbb{P}^{-}}^{p}$ continuity of the Koopman operator $T$, the following equalities hold both $\mathbb{P}$-a.s. and in $L_{\mathbb{P}}^{p}$ (with $p \geq 1):$ if $Y \in L_{\mathbb{P}}^{p}$

$$
T^{k} E_{-\infty} Y=T^{k} \lim _{j} E_{-j+k} Y=\lim _{j} T^{k} E_{-j+k} Y=\lim _{j} E_{-j} T^{k} Y=E_{-\infty} T^{k} Y .
$$

Let us give now a result that, in the context of the present discussion, will allow us to give a further characterization of the regularity condition (29) (see Corollary 5.14) and is of interest by itself. We will use also this result later to prove part of the statement of Theorem 2 below.

Proposition 5.12. The equality

$$
\lim _{n} \frac{1}{n} \sum_{k=0}^{n-1}\left|E_{0} X_{k}\right|^{2}=\lim _{n}\left\|E_{0} X_{n}\right\|_{\mathbb{P}, 2}^{2}
$$

holds $\mathbb{P}$-a.s.

Proof. $E_{0} T$ is a positive Dunford-Scwhartz operator and therefore it is pointwise and mean ergodic ([16], Theorem 8.24 and pp. 217-218): for every $Y \in L_{\mathbb{P}}^{p}(p \geq 1)$,

$$
\frac{1}{n} \sum_{k=0}^{n-1} E_{0} T^{k} Y=E_{0} E[Y \mid \mathcal{T}]=E Y,
$$

$\mathbb{P}$-a.s. and in $L_{\mathbb{P}}^{p}$, where $\mathcal{T}$ is the invariant sigma algebra of $T$ and the second equality follows from the assumption that $T$ is ergodic (we can identify the limit function using the continuity of the operator $E_{0}$ in $L_{\mathbb{P}}^{p}$ and the mean ergodic theorem for Koopman operators).

Now notice that, since for every $Z \in L_{\mathbb{P}}^{2}$ and $(n, j) \in \mathbb{N} \times \mathbb{N},\left|E_{0} T^{n+j} Z\right|^{2}=\left|\left(E_{0} T\right)^{n+j} Z\right|^{2} \leq$ $E_{0} T^{n}\left|E_{0} T^{j} Z\right|^{2}, \mathbb{P}$-a.s., the process $\left(F_{n}\right)_{n \in \mathbb{N}}$ specified by

$$
F_{n}:=\sum_{k=0}^{n-1} E_{0} T^{k}\left|X_{0}\right|^{2}-\sum_{k=0}^{n-1}\left|E_{0} T^{k} X_{0}\right|^{2}
$$

is nonnegative and superadditive ([18], p. 146., or [1]).

An application of [18], Theorem 5.3, together with the Dunford-Schwartz pointwise ergodic theorem, implies that there exists a (nonnegative) function $Y \in L_{\mathbb{P}}^{1}$ such that 
1. $E Y=\lim _{n} E F_{n} / n$,

2. For every $n \in \mathbb{N}$

$$
F_{n} \leq G_{n}:=\sum_{k=0}^{n-1} E_{0} T^{k} Y
$$

$\mathbb{P}$-a.s., and

3. $\lim _{n} F_{n} / G_{n}=1, \mathbb{P}$-a.s. on $\bigcup_{k \in \mathbb{N}}\left[E_{0} T^{k} Y>0\right]$ (either this set or its complement has probability one in this case, but this is not assumed for what follows along this proof).

It follows from these observations (note also that $F_{n}=0, \mathbb{P}$-a.s., on $\bigcap_{k \in \mathbb{N}}\left[E_{0} T^{k} Y=0\right]$ ) and another application of the Dunford-Schwartz ergodic theorem that

$$
\lim _{n} \frac{1}{n} \sum_{k=0}^{n-1}\left|E_{0} T^{k} X_{0}\right|^{2}=E\left[\left|X_{0}\right|^{2}-Y\right]
$$

$\mathbb{P}_{\text {-a.s. }}{ }^{9}$

To actually show that $E Y=\left\|X_{0}\right\|_{\mathbb{P}, 2}^{2}-\lim _{n}\left\|E_{0} T^{n} X_{0}\right\|_{\mathbb{P}, 2}^{2}$ we first notice that

$$
E Y=\lim _{n} \frac{E F_{n}}{n}=\lim _{n}\left(\left\|X_{0}\right\|_{\mathbb{P}, 2}^{2}-\frac{1}{n} \sum_{k=0}^{n-1}\left\|E_{0} T^{k} X_{0}\right\|_{\mathbb{P}, 2}^{2}\right)
$$

$\mathbb{P}$-a.s., and that by Jensen's inequality

$$
\left\|E_{0} T^{k+1} X_{0}\right\|_{\mathbb{P}, 2}^{2}:=E\left|E_{0} T^{k+1} X_{0}\right|^{2} \leq E\left|E_{0} T^{k} X_{0}\right|^{2}=:\left\|E_{0} T^{k} X_{0}\right\|_{\mathbb{P}, 2}^{2} .
$$

Thus the sequence $\left(\left\|E_{0} T^{k} X_{0}\right\|_{\mathbb{P}, 2}^{2}\right)_{k \in \mathbb{N}}$ is decreasing. It follows that

$$
\lim _{n} \frac{1}{n} \sum_{k=0}^{n-1}\left\|E_{0} T^{k} X_{0}\right\|_{\mathbb{P}, 2}^{2}=\lim _{n}\left\|E_{0} T^{n} X_{0}\right\|_{\mathbb{P}, 2}^{2}
$$

Remark 5.13. Using exactly the same argument as in the proof of Proposition 5.12, one can further prove the $\mathbb{P}$-a.s. convergence

$$
\lim _{n} \frac{1}{n} \sum_{k=0}^{n-1}\left|E_{0} T^{k} X_{0}\right|^{\beta}=\lim _{n} E\left|E_{0} X_{n}\right|^{\beta}
$$

\footnotetext{
${ }^{9}$ An alternative argument is the following: by (33), the invariant sigma algebra of the operator $E_{0} T$ is trivial, and it follows by an application of Corollary 1 in [1] with $s_{k}^{\prime}=k$ that $F_{n} / n \rightarrow_{n} E Y, \mathbb{P}$-a.s. The pointwise ergodicity of $E_{0} T$ (i.e., a novel application of (33)) gives (35).
} 
for every $1 \leq \beta \leq p$, provided that $X_{0} \in L_{\mathbb{P}}^{p}$. A similar argument proves the same conclusion when $0 \leq \beta<1 \leq p$ (in this case the process $\left(\sum_{k=0}^{n-1}\left|E_{0} T^{k} X_{0}\right|^{\beta}\right)_{n \in \mathbb{N}^{*}}$ is superadditive). ${ }^{10}$

More generally, an easy adaptation of the first part of the arguments in this proof (using the same results from [18]) proves that if $L$ is a positive Dunford-Schwartz operator (or any conservative positive contraction that is also pointwise ergodic) and $\varphi:[0, \infty) \rightarrow[0, \infty)$ is a nonnegative measurable function for which $\varphi \circ L X \leq L(\varphi \circ X), \mathbb{P}$-a.s. for a given nonnegative $X \in L_{\mathbb{P}}^{p}$ $(p \geq 1)$, then

$$
\frac{1}{n} \sum_{k=0}^{n-1} \varphi\left(L^{k} X\right)
$$

converges $\mathbb{P}$-a.s.

The following is an obvious consequence of Proposition 5.12.

Corollary 5.14. The process $\left(X_{k}\right)_{k \in \mathbb{Z}}$ is regular (Definition 5.10) if and only if

$$
\lim _{n} \frac{1}{n} \sum_{k=0}^{n-1}\left|E_{0} X_{k}\right|^{2}=0,
$$

$\mathbb{P}$-a.s.

Let us now introduce is the "averaged-frequency" version of Lemma 5.5, which will be the building block for the proof of Theorem 2 .

Lemma 5.15. If $\mathcal{B}_{0}=\{\varnothing,[0,2 \pi)\}$ is the trivial sigma algebra and $0 \leq r \leq \infty$ is fixed, the random function $(\theta, \omega) \mapsto V_{r, n}(\theta, \omega)$ defined by (19) (where $V_{\infty, n}(\theta, \omega):=0$ if $\left.\theta \notin I\right)$ converges in the quenched sense with respect to $\mathcal{B}_{0} \otimes \mathcal{F}_{0}$ (see Section 3.2), as $n \rightarrow \infty$, to the function $\left(\theta, \omega^{\prime}\right) \mapsto B_{r}\left(\theta, \omega^{\prime}\right)$ defined by (22) (where $B\left(\theta, \omega^{\prime}\right)=0$ if $\left.\theta \notin I\right)$. Equivalently, there exists $\Omega_{0} \subset \Omega$ with $\mathbb{P} \Omega_{0}=1$ such that for every $\omega \in \Omega_{0}, V_{r, n} \Rightarrow_{n} B_{r}$ under $\lambda \times \mathbb{P}_{\omega}$.

Proof. This follows at once from Corollary 6.2 in Section 6.

We will use Lemma 5.15 in order to prove Theorem 2 below. We will also need the following lemma, which is essentially the same as Lemma 5.6 except that, just as in Lemma 5.15, the processes involved are seen as random elements whose domain is the product space $[0,2 \pi) \times \Omega$, and the notion of quenched convergence is understood with respect to $\mathcal{B}_{0} \otimes \mathcal{F}_{0}$. The proof is exactly as that of Lemma 5.6.

${ }^{10}$ There is an additional detail in this case: to prove that the increasing, Cesàro-convergent sequence $\left(E\left|E_{0} T^{n} X\right|^{\beta}\right)_{n \in \mathbb{N}}$ is bounded (i.e., convergent) note for instance that, by Hölder's inequality and Jensen's inequality

$$
\left\|E_{0} T^{n} X\right\|_{\mathbb{P}, \beta} \leq\left\|E_{0} T^{n} X\right\|_{\mathbb{P}, p} \leq\|X\|_{\mathbb{P}, p}
$$

for all $n \in \mathbb{N}$ (it is actually a short exercise to prove that an unbounded and increasing sequence is not Cesàro convergent: its Cesàro averages also diverge to $\infty$ ). 
Lemma 5.16. With the notation introduced in Section 3.2 and in (17)-(21) (see also Remark 3.3), and taking $\mathcal{B}_{0}:=\{\varnothing,[0,2 \pi)\}$, assume that

$$
\lim _{r} \limsup _{n} \tilde{E}_{0}\left[\frac{1}{n} \max _{1 \leq k \leq n}\left|S_{k}(\theta, \omega)-E_{0} S_{k}(\theta, \omega)-M_{r, k}(\theta, \omega)\right|^{2}\right]=0
$$

$\mathbb{P}$-a.s. Then the random function $(\theta, \omega) \mapsto W_{n}(\theta, \omega)$ specified by (20) converges in the quenched sense as $n \rightarrow \infty$, with respect to $\mathcal{B}_{0} \otimes \mathcal{F}_{0}$, to the random function $\left(\theta, \omega^{\prime}\right) \mapsto B\left(\theta, \omega^{\prime}\right)$ specified by (23) $(B(\theta, \omega)=0$ if $\theta \notin I)$. Equivalently, there exists $\Omega_{0} \subset \Omega$ with $\mathbb{P} \Omega_{0}=1$ such that for every $\omega \in \Omega_{0}, W_{n} \Rightarrow_{n} B$ under $\lambda \times \mathbb{P}_{\omega}$.

\subsection{Main results}

We will prove that the hypotheses in Lemma 5.16 hold under the general setting established in Section 3. Let us state the respective result in terms of the regular conditional measures associated to $E_{0}$ :

Theorem 2. There exists $\Omega_{0} \subset \Omega$ with $\mathbb{P} \Omega_{0}=1$ such that for every $\omega_{0} \in \Omega_{0}$ the random functions

$$
W_{n}:\left([0,2 \pi) \times \Omega, \mathcal{B} \otimes \mathcal{F}, \lambda \times \mathbb{P}_{\omega_{0}}\right) \rightarrow D[[0, \infty), \mathbb{C}]
$$

specified by (20) (see also Remark 5.4) converge in distribution, as $n \rightarrow \infty$, to the random function

$$
B:\left([0,2 \pi) \times \Omega^{\prime}, \mathcal{B} \otimes \mathcal{F}^{\prime}, \lambda \times \mathbb{P}^{\prime}\right) \rightarrow D[[0, \infty), \mathbb{C}]
$$

specified by (23) (with $B(\theta, \omega)=0$ if $\theta \notin I)$. If (29) holds, then the non-centered cadlag functions (21) satisfy the same conclusion.

Note that, by the argument preceding Remark 2.1, this theorem implies Proposition 2.1 in [20]. This theorem will be proved in Section 7.

We will now present the cases in which the estimate (24) is proved along this paper, the notation introduced in the lemmas above is kept here.

To present our results start by considering the following conditions of weak dependence

(a) The generalized Hannan condition, given by

$$
\sum_{n \geq 0}\left\|\mathcal{P}_{0}\left(X_{n+1}-X_{n}\right)\right\|_{\mathbb{P}, 2}<\infty
$$


which is clearly a "weak" version of the Hannan condition ${ }^{11}$

$$
\sum_{n}\left\|\mathcal{P}_{0} X_{n}\right\|_{\mathbb{P}, 2}<\infty
$$

Note, on the other side, that (37) is the same as (38) for the (stationary) process $\left(Y_{n}\right)_{n}$ specified by

$$
Y_{n}:=X_{n}-X_{n-1}
$$

(b) The Maxwell and Woodroofe condition for a fixed frequency. Which states that, for $\theta \in$ $[0,2 \pi)$,

$$
\sum_{k=1}^{\infty} \frac{\left\|E_{0} S_{k}(\theta)\right\|_{\mathbb{P}, 2}}{k^{3 / 2}}<\infty .
$$

Condition (39) is a "rotated" version of the Maxwell and Woodroofe condition

$$
\sum_{k=1}^{\infty} \frac{\left\|E_{0} S_{k}\right\|_{\mathbb{P}, 2}}{k^{3 / 2}}<\infty
$$

Conditions (37) and (40) are logically independent, see [13], ${ }^{12}$ and they imply the existence of martingale approximations allowing us to prove the following theorem:

Theorem 3. With the notation introduced in lemmas 5.5 and 5.6, and assuming (37) or (39), if $e^{2 i \theta} \notin \operatorname{Spec}_{p}(T)$, then (20) converges in the quenched sense, as $n \rightarrow \infty$, to

$$
\omega^{\prime} \mapsto \frac{\sigma(\theta)}{\sqrt{2}}\left(\operatorname{Re}(B)\left(\omega^{\prime}\right)+i \operatorname{Im}(B)\left(\omega^{\prime}\right)\right) .
$$

If (39) holds, (also) the non-centered cadlag functions (21) converge in the quenched sense, as $n \rightarrow \infty$, to $(41)$.

It is important to point out the following distinction implicit in the statement of Theorem 3: when condition (37) holds, Theorem 3 tells us that, for every $\theta$ with $e^{2 i \theta} \notin \operatorname{Spec}_{p}(T)$, and in

${ }^{11}$ To see that (37) is strictly weaker than (38) consider the process

$$
X_{k}:=\sum_{j \in \mathbb{N}^{*}} \frac{1}{j} x_{k-j},
$$

where $\left(x_{j}\right)_{j \in \mathbb{Z}}$ are the coordinate functions in $\mathbb{R}^{\mathbb{Z}}$, seen as an i.i.d. centered sequence in $L^{2}, T$ is the left shift, and $\mathcal{F}_{0}=\sigma\left(x_{k}\right)_{k \leq 0}$.

${ }^{12}$ More precisely, the results stated in [13] give that (37) is not sufficient for (40). To prove that (37) is not necessary for (40) consider the process constructed in [13], and strengthen the assumption " $N_{k+1}>N_{k}$ " to " $N_{k+1}>N_{k}+1$ ". Following the arguments in that paper, it is easy to see that (37) and (38) are equivalent for the process under consideration, and the proof of independence can be carried over by substituting, in the first counterexample on [13], " $N_{k}=k$ " by " $N_{k}=3 k$ ". 
particular for $\lambda$-a.e. $\theta$ (see (11)), (20) converges in the quenched sense, whereas the conclusion under the Maxwell and Woodroofe condition (39) is that for a given $\theta$ with $e^{2 i \theta} \notin \operatorname{Spec}_{p}(T)$, (20) and (21) converge in the quenched sense (to the same limit function) if (39) holds.

It is therefore desirable to give a criterion implying the fulfillment of (39) for $\lambda$-a.e. $\theta$. This is the content of the following result.

Theorem 4. Iffor some $\beta>1$

$$
\sum_{k=1}^{\infty}(\log k)^{\beta} \frac{\left\|E_{0} X_{k}\right\|_{\mathbb{P}, 2}^{2}}{k}<\infty
$$

then (39) holds for $\lambda$-a.e. $\theta$. In particular (42) implies that (21) (and (20)) converges in the quenched sense, as $n \rightarrow \infty$, for $\lambda$-a.e. $\theta$, as specified in Theorem 3 .

Remark 5.17. One may naturally ask if, just as in the case of Theorem 2, the verification of (29) together with the quenched convergence of (20) for a given $\theta$ (or for $\lambda$-a.e. $\theta$ ) are sufficient conditions for the quenched convergence of (21) as $n \rightarrow \infty$.

The actual answer is no: consider one more time $\left(x_{j}\right)_{j \in \mathbb{Z}},\left(\mathcal{F}_{j}\right)_{j \in \mathbb{Z}}, T$ as in the footnote following (37) and note again that any (centered) linear process

$$
X_{k}=\sum_{j \in \mathbb{N}} a_{j} x_{k-j}
$$

with $\left(a_{j}\right)_{j \in \mathbb{N}} \in l^{1}(\mathbb{N})\left(\left(x_{j}\right)_{j \in \mathbb{N}}\right.$ i.i.d., $\left.E x_{0}=0, E\left|x_{0}\right|^{2}=1\right)$ satisfies (38) and therefore also (37). Thus the conclusion of Theorem 3 holds for (20) when $\left(X_{k}\right)_{k \in \mathbb{Z}}$ is given by (43), $\left(a_{j}\right)_{j \in \mathbb{N}} \in l^{1}(\mathbb{N})$ and $\theta \in(0,2 \pi) \backslash\{\pi\}$ (see Corollary 5.19 below).

Such a process is necessarily regular because, by Kolmogorov's zero-one law, $E_{-\infty} Z=E Z$, $\mathbb{P}$-a.s. for every $Z \in L_{\mathbb{P}}^{1}$ in this case but, as shown in [3], the coefficients $\left(a_{k}\right)_{k \in \mathbb{N}}$ can be chosen in such a way that, for every $\theta \in[0,2 \pi), S_{n}(\theta) / \sqrt{n}$ admits no limit in distribution under $\mathbb{P}_{\omega}$ for $\mathbb{P}_{-}$ a.e. $\omega$. This is of course an obstruction (consider $t=1$ ) to the quenched convergence, as $n \rightarrow \infty$, of (21) to (41) or to any random function that is continuous at 1 with probability one, but we can discard convergence to any other random function in $D[[0, \infty), \mathbb{C}]$ considering the identity $U_{n}(\theta, t)=(\lfloor n t\rfloor / n)^{1 / 2} U_{\lfloor n t\rfloor}(\theta, 1)\left(U_{n}\right.$ is given by $\left.(21)\right)$ and the general arguments behind the observation 2. in Section 4. See for instance [7], p. 138.

These considerations support the claim that (even) under the assumptions presented along this paper, including the regularity condition (29), the quenched functional convergence with respect to $\mathcal{F}_{0}$ of $S_{n}(\theta) / \sqrt{n}$ for $\lambda$-a.e. fixed $\theta$ as $n \rightarrow \infty$ is a conclusion strictly stronger than that of the quenched functional convergence with respect to $\{\varnothing,[0,2 \pi)\} \otimes \mathcal{F}_{0}$ of the same process seen as a process parametrized by $(\theta, \omega)$. More explicitly, if $\left(X_{k}\right)_{k \in \mathbb{Z}}$ is the process constructed in [3] then, by Theorem 2, $(\theta, \omega) \mapsto U_{n}(\theta, \omega)$ converges in the quenched sense with respect to $\{\varnothing,[0,2 \pi)\} \otimes \mathcal{F}_{0}$ to $\left(\theta, \omega^{\prime}\right) \mapsto B\left(\theta, \omega^{\prime}\right)$, and we have just mentioned that (21) does not converge (in $D[[0, \infty), \mathbb{C}]$ ) in the quenched sense with respect to $\mathcal{F}_{0}$ for any fixed $\theta \in[0,2 \pi)$, so that we cannot obtain the conclusion of Theorem 3 for (21) from the regularity condition (29) in spite that 
the (full) conclusion of Theorem 2 is true for a process satisfying this condition. The question of whether this is also the case for (20) seems to be still open, as hinted already in Remark 5.9.

Finally, note that these kind of considerations show that one cannot deduce the fulfillment of (39) for $\lambda$-a.e. $\theta$, not even for any given fixed $\theta$, from the fulfillment of (37) only. ${ }^{13}$ In particular, (42) does not follow from (37).

Remark 5.18. It is also convenient to point out the relation between (42) and (29): first, it clearly follows from (42) that

$$
\sum_{k=1}^{\infty} \frac{\left\|E_{0} X_{k}\right\|_{\mathbb{P}, 2}^{2}}{k}<\infty
$$

and this, together with Kronecker's lemma, implies that under (42)

$$
\lim _{n} \frac{1}{n} \sum_{k=0}^{n-1}\left\|E_{0} X_{k}\right\|_{\mathbb{P}, 2}^{2}=0,
$$

which is the same as (29) because $\left(\left\|E_{0} X_{k}\right\|_{\mathbb{P}, 2}^{2}\right)_{k \in \mathbb{N}}$ is decreasing.

On the other side, (29) does not imply (42) because, as we have seen in Remark 5.17, there exist processes satisfying (29) that do not satisfy the conclusion of Theorem 3. Thus, (42) is strictly stronger than (29).

Collecting our results, the following question deserves to be pointed out: is there $0 \leq \beta \leq 1$ such that (42) holds for a process that does not satisfy the conclusion of Theorem 3? (with and without the statement of convergence for (21)). And if the answer is "no", what conditions "in between" (44) and (29) break down the conclusion of Theorem 3?

To finish this section, it is worth to further specify a case in which the set of frequencies where the asymptotic distribution is as in (41) can be easily described. To motivate the following result, recall that $T$ is weakly mixing if and only if $\operatorname{Spec}_{p}(T)=\{1\}$ (see [21], Section 8 for a review of this and other related facts).

Now, as a subgroup of $\mathbb{T}, \operatorname{Spec}_{p}(T)$ is finite (actually: closed) if and only if there exists $m \in \mathbb{N}^{*}$ such that

$$
\operatorname{Spec}_{p}(T):=\left\{e^{2 \pi k i / m}\right\}_{k=0}^{m-1} .
$$

In other words, $\operatorname{Spec}_{p}(T)$ is finite if and only it it consists of the points in the unit circle given by the rational rotations by an angle of $2 \pi / m$ or, what is the same, by the $m$ th roots of unity.

Corollary 5.19. Assume that $\operatorname{Spec}_{p}(T)$ is finite and its elements are the mth roots of unity. Under the hypotheses in Theorem 3, (20) converges in the quenched sense as $n \rightarrow \infty$ to (41) for all $\theta \in[0,2 \pi)$ such that $e^{2 i m \theta} \neq 1$. If $T$ is in particular weakly mixing, (41) describes the asymptotic quenched limit of (20) for all $\theta \neq 0, \pi$. The same conclusions hold for (21) when (39) holds.

\footnotetext{
${ }^{13}$ Except perhaps, though one would not expect such thing, if $e^{2 i \theta} \in \operatorname{Spec}_{p}(T)$ and $\theta \neq 0$ are conditions imposed on $\theta$. The case $\theta=0$ being covered by [13] as specified above, and the case $e^{2 i \theta} \notin \operatorname{Spec}_{p}(T)$ being covered by our considerations.
} 
Proof. Immediate from (45), Theorem 3 and Theorem 4.

Remark 5.20. Of course, the conclusion of Theorem 3 is also true for the weakly mixing case under (42) $(\beta>1)$, but our proof in this case losses track of the ( $\lambda$-measure one) set of frequencies $\theta$ where (39) holds.

\section{Martingale case}

Since our proofs are based on martingale approximations we will study, in this section, the asymptotic distributions of (19) for the case in which $D_{r, 0}(\theta)$ is replaced by an abstract martingale difference $D_{0}(\theta)$.

The result is Theorem 5. The martingale case on the product space ("averaged frequency" case) follows from this one via some of the general results presented in this paper, and it is the content of Corollary 6.2.

Theorem 5. Under the setting in Section 3.1, and with the notation in Lemma 5.5, given $\theta \in$ $[0,2 \pi)$ such that $e^{2 i \theta} \notin \operatorname{Spec}_{p}(T)$, assume that $D_{0}(\theta) \in L_{\mathbb{P}}^{2}\left(\mathcal{F}_{0}\right) \ominus L_{\mathbb{P}}^{2}\left(\mathcal{F}_{-1}\right)$ is given, and define

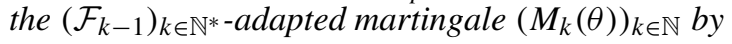

$$
M_{n}(\theta):=\sum_{k=0}^{n-1} T^{k} D_{0}(\theta) e^{i k \theta}
$$

for all $n \in \mathbb{N}$. Then the sequence $\left(V_{k}(\theta)\right)_{k \in \mathbb{N}^{*}}$ of random elements of $D[[0, \infty), \mathbb{C}]$ defined by

$$
V_{n}(\theta)(t):=M_{\lfloor n t\rfloor}(\theta) / \sqrt{n}
$$

for every $n \in \mathbb{N}^{*}$, converges in the quenched sense with respect to $\mathcal{F}_{0}$ to the random function $B(\theta): \Omega^{\prime} \rightarrow D[[0, \infty), \mathbb{C}]$ given by

$$
B(\theta)\left(\omega^{\prime}\right)=\left[E\left|D_{0}(\theta)\right|^{2} / 2\right]^{1 / 2}\left(\operatorname{Re}(B)\left(\omega^{\prime}\right)+i \operatorname{Im}(B)\left(\omega^{\prime}\right)\right) .
$$

Remark 6.1. Before proceeding to the proof it is worth noticing the following: the conclusion of Theorem 1, specialized to this case, is a statement about the asymptotic distribution of the random variables $V_{n}(\theta)(1)$. By Lemma A.6 in the Appendix and the orthogonality under $E_{0}$ of $\left(T^{k} D_{0}(\theta)\right)_{k \in \mathbb{N}},^{14}$

$$
E\left[\left|D_{0}(\theta)\right|^{2}\right]=\lim _{n} \frac{1}{n} \sum_{k=1}^{n-1} E_{0} T^{k}\left|D_{0}(\theta)\right|^{2}=\lim _{n} \frac{1}{n} E_{0}\left|M_{n}(\theta)-E_{0} M_{n}(\theta)\right|^{2},
$$

and therefore the equality (16) is certainly verified in this case.

\footnotetext{
${ }^{14}$ Note that if $(k, r) \in \mathbb{N} \times \mathbb{N}^{*}$ is given then, since $T^{r} D_{0}(\theta) \in L_{\mathbb{P}}^{2}\left(\mathcal{F}_{r}\right) \ominus L_{\mathbb{P}}^{2}\left(\mathcal{F}_{r-1}\right)$, $E_{0}\left[T^{k} D_{0}(\theta) T^{k+r} \overline{D_{0}(\theta)}\right]=E_{0}\left[T^{k} D_{0}(\theta) E_{k} T^{k+r} \overline{D_{0}(\theta)}\right]=E_{0} T^{k}\left[D_{0}(\theta) E_{0} T^{r} \overline{D_{0}(\theta)}\right]=0$,

$\mathbb{P}$-a.s.
} 
Proof of Theorem 5. Let us start by sketching the argument of the proof: we will see that for $\theta$ as specified, there exists $\Omega_{\theta} \subset \Omega$ with $\mathbb{P} \Omega_{\theta}=1$ such that for every $\omega \in \Omega_{\theta}$ the following holds:

(a) The sequence of random functions $\left(V_{n}(\theta)\right)_{n}$ in $D[[0, \infty), \mathbb{C}]$ is tight with respect to $\mathbb{P}_{\omega}$. To prove this, we will actually prove the convergence in distribution of both the real and imaginary parts of $\left(V_{n}(\theta)\right)_{n}$ to a Brownian motion via Theorem A.14.

(b) The finite dimensional asymptotic distributions under $\mathbb{P}_{\omega}$ of $\left(V_{n}(\theta)\right)_{n}$ converge to those of two independent standard motions with the rescaling $E\left[\left(D_{0}(\theta)\right)^{2}\right]^{1 / 2} / \sqrt{2}$. For this, we will proceed via the Cramér-Wold theorem, using some of the results already presented.

We go now to the details: first, we will assume, making it explicit only when necessary, that $E_{0}$ is the version of $E\left[\cdot \mid \mathcal{F}_{0}\right]$ given by integration with respect to the decomposing probability measures $\left\{\mathbb{P}_{\omega}\right\}_{\omega \in \Omega}$.

Now denote, for every $k \in \mathbb{N}$

$$
D_{k}(\theta):=T^{k} D_{0}(\theta) .
$$

Let $\Omega_{\theta, 1}^{\prime}$ be the set of probability one guaranteed by Lemma A.9 for the case $Y=D_{0}(\theta)$. By Remark 2.3, there exists a set $\Omega_{\theta, 1}$ with $\mathbb{P} \Omega_{\theta, 1}=1$ such that for every $\omega \in \Omega_{\theta, 1}$

$$
\lim _{n} \frac{1}{n} \sum_{k=0}^{n-1} E_{k-1}\left(z \cdot\left(D_{k}(\theta) e^{i k \theta}\right)\right)^{2}=\frac{|z|^{2}}{2} E\left|D_{0}(\theta)\right|^{2}
$$

$\mathbb{P}_{\omega}$-a.s. for all $z \in \mathbb{C}$.

For such $\omega$ 's the first hypothesis of Theorem A.14 is verified by the triangular arrays $\left(\operatorname{Re}\left(M_{k}(\theta) / \sqrt{n}\right)\right)_{1 \leq k \leq n}$ and $\left(\operatorname{Im}\left(M_{k}(\theta) / \sqrt{n}\right)\right)_{1 \leq k \leq n}\left(n \in \mathbb{N}^{*}\right)$ with respect to $\mathbb{P}_{\omega}$, because they arise from the particular choices $z=1$ and $z=i$, respectively.

To verify the second hypothesis in Theorem A.14, we start from the $\mathbb{P}$-a.s. inequality

$$
\begin{aligned}
& E_{0}\left[\frac{1}{n} \sum_{k=0}^{n-1}\left(\left(\operatorname{Re}\left(D_{k}(\theta) e^{i k \theta}\right)\right)^{2} I_{\left[\left|\operatorname{Re}\left(D_{k}(\theta) e^{i k \theta}\right)\right| \geq \epsilon \sqrt{n}\right]}+\left(\operatorname{Im}\left(D_{k}(\theta) e^{i k \theta}\right)\right)^{2} I_{\left[\left|\operatorname{Im}\left(D_{k}(\theta) e^{i k \theta}\right)\right| \geq \epsilon \sqrt{n}\right]}\right)\right] \\
& \leq E_{0}\left[\frac{1}{n} \sum_{k=0}^{n-1}\left|D_{k}(\theta)\right|^{2} I_{\left[\left|D_{k}(\theta)\right| \geq \epsilon \sqrt{n}\right]}\right] .
\end{aligned}
$$

Now, given $\eta>0$ there exists $N \geq 0$ such that $\mu_{N}:=E\left[\left|D_{0}(\theta)\right|^{2} I_{\left[\left|D_{0}(\theta)\right|^{2} \geq \epsilon^{2} N\right]}\right]<\eta$, and therefore

$$
\begin{aligned}
& \limsup _{n} \frac{1}{n} \sum_{k=0}^{n-1} E_{0} T^{k}\left[\left|D_{0}(\theta)\right|^{2} I_{\left[\left|D_{0}(\theta)\right|^{2} \geq \epsilon^{2} n\right]}\right] \\
& \quad \leq \limsup _{n} \frac{1}{n} \sum_{k=0}^{n-1} E_{0} T^{k}\left[\left|D_{0}(\theta)\right|^{2} I_{\left[\left|D_{0}(\theta)\right|^{2} \geq \epsilon^{2} N\right]}\right]=\mu_{N} \leq \eta
\end{aligned}
$$

over a set $\Omega_{\theta, \epsilon, \eta}$ with $\mathbb{P} \Omega_{\theta, \epsilon, \eta}=1$, where we made use of Corollary A.6. Without loss of generality, (50) holds for all $\omega \in \Omega_{\theta, \epsilon, \eta}$. 
Denote by $Z_{n}^{\epsilon}$ the random variable at the left-hand side of the inequality (50) and note that, if we define

$$
\Omega_{\theta, 2}=\bigcap_{\epsilon>0, \eta>0} \Omega_{\theta, \epsilon, \eta}
$$

where the intersection runs over rational $\epsilon, \eta$, then $\mathbb{P} \Omega_{\theta, 2}=1$, and for every $\epsilon>0$ and every $\omega \in \Omega_{\theta, 2}$

$$
\lim _{n} Z_{n}^{\epsilon}(\omega)=0
$$

or, what is the same, for all $\omega \in \Omega_{\theta, 2}$

$$
\frac{1}{n} \sum_{k=0}^{n-1}\left(\left(\operatorname{Re}\left(D_{k}(\theta) e^{i k \theta}\right)\right)^{2} I_{\left[\left|\operatorname{Re}\left(D_{k}(\theta) e^{i k \theta}\right)\right| \geq \epsilon \sqrt{n}\right]}+\left(\operatorname{Im}\left(D_{k}(\theta) e^{i k \theta}\right)\right)^{2} I_{\left[\left|\operatorname{Im}\left(D_{k}(\theta) e^{i k \theta}\right)\right| \geq \epsilon \sqrt{n}\right]}\right)
$$

goes to 0 in $L_{\mathbb{P}_{\omega}}^{1}$ as $n \rightarrow \infty$.

Thus, if $\Omega_{\theta, 3}$ is a set of probability one such that $\left(\operatorname{Re}\left(M_{k}(\theta)\right)\right)_{k \in \mathbb{N}^{*}}$ and $\left(\operatorname{Im}\left(M_{k}(\theta)\right)\right)_{k \in \mathbb{N}^{*}}$ is a

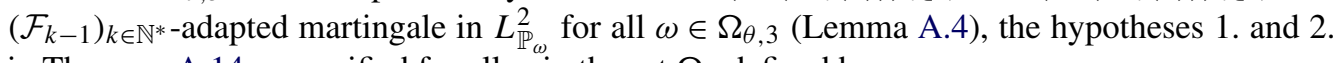
in Theorem A.14 are verified for all $\omega$ in the set $\Omega_{\theta}$ defined by

$$
\Omega_{\theta}:=\bigcap_{k=1}^{3} \Omega_{\theta, k}
$$

Since $\mathbb{P} \Omega_{\theta}=1$ this finishes the proof of (a).

To prove (b) we will show that for any given $n \in \mathbb{N}$, any $\omega \in \Omega_{\theta}$, and any $0 \leq t_{1} \leq \cdots \leq t_{n}$, the $\mathbb{C}^{n}=\mathbb{R}^{2 n}$-valued process

$$
\left(V_{n}(\theta)\left(t_{1}\right), V_{n}(\theta)\left(t_{2}\right)-V_{n}(\theta)\left(t_{1}\right), \ldots, V_{n}(\theta)\left(t_{n}\right)-V_{n}(\theta)\left(t_{n-1}\right)\right)
$$

has the same asymptotic distribution as

$$
\begin{aligned}
& \mathbf{B}^{\theta}\left(t_{1}, \ldots, t_{n}\right) \\
& \quad:=\left[E\left|D_{0}(\theta)\right|^{2} / 2\right]^{1 / 2}\left(\Delta_{0} \operatorname{Re}(B), \Delta_{0} \operatorname{Im}(B), \Delta_{1} \operatorname{Re}(B), \Delta_{1} \operatorname{Im}(B), \ldots, \Delta_{n-1} \operatorname{Im}(B)\right)
\end{aligned}
$$

under $\mathbb{P}_{\omega}$ where $\Delta_{k} F:=F\left(t_{k+1}\right)-F\left(t_{k}\right)$ and $t_{0}=0$ and therefore, by the mapping theorem ([7], Theorem 2.7), the finite dimensional asymptotic distributions of $V_{n}(\theta)$ under $\mathbb{P}_{\omega}$ and those of (48) under $\mathbb{P}^{\prime}$ are the same.

For simplicity, we will assume $n=2$. The argument generalizes easily to an arbitrary $n \in \mathbb{N}$.

Our goal is thus to prove that for all $\omega \in \Omega_{\theta}$ and all $0 \leq s \leq t$ the asymptotic distribution of

$$
\mathbf{V}_{n}^{\theta}(s, t):=\left(V_{n}(\theta)(s), V_{n}(\theta)(t)-V_{n}(\theta)(s)\right)
$$


(a $\mathbb{C}^{2}=\mathbb{R}^{4}$-valued process) is the same under $\mathbb{P}_{\omega}$ as that of

$$
\begin{aligned}
\mathbf{B}^{\theta}(s, t):= & {\left[E\left|D_{0}(\theta)\right|^{2} / 2\right]^{1 / 2} } \\
& \times(\operatorname{Re}(B)(s), \operatorname{Im}(B)(s), \operatorname{Re}(B)(t)-\operatorname{Re}(B)(s), \operatorname{Im}(B)(t)-\operatorname{Im}(B)(s))
\end{aligned}
$$

under $\mathbb{P}^{\prime}$.

To prove the convergence in distribution of (54) to (55), we will use the Cramér-Wold theorem. This is, we will see that for any $\omega \in \Omega_{\theta}$, any $0 \leq s \leq t$, and any

$$
\mathbf{u}=\left(a_{1}, a_{2}, b_{1}, b_{2}\right) \in \mathbb{R}^{4}
$$

the asymptotic distribution under $\mathbb{P}_{\omega}$ of the stochastic process $\left(U_{n}\right)_{n \in \mathbb{N}^{*}}$ defined by

$$
U_{n}:=\mathbf{u} \cdot \mathbf{V}_{n}^{\theta}(s, t)
$$

is that of a normal random variable with variance

$$
\sigma_{\mathbf{u}, s, t}^{2}(\theta):=\frac{E\left[\left|D_{0}(\theta)\right|^{2}\right]}{2}\left(\left(a_{1}^{2}+a_{2}^{2}\right) s+\left(b_{1}^{2}+b_{2}^{2}\right)(t-s)\right) .
$$

To do so, we will verify the hypotheses of Theorem A.13. Fix $\mathbf{u}$ as above and note that

$$
U_{n}=\sum_{k=0}^{\lfloor n s\rfloor} \eta_{n k}\left(a_{1}, a_{2}\right)+\sum_{k=\lfloor n s\rfloor+1}^{\lfloor n t\rfloor} \eta_{n k}\left(b_{1}, b_{2}\right)
$$

where

$$
\eta_{n k}\left(x_{1}, x_{2}\right)=\frac{1}{\sqrt{n}}\left(x_{1}, x_{2}\right) \cdot e^{i k \theta} T^{k} D_{0}(\theta) .
$$

By the construction of $\Omega_{\theta}$, for every $0 \leq r$, every $x_{1}, x_{2}$ and every $\omega \in \Omega_{\theta},\left(\eta_{n k}\left(x_{1}\right.\right.$, $\left.\left.x_{2}\right)\right)_{0 \leq k \leq\lfloor n r\rfloor}$ is a triangular array of $\left(\mathcal{F}_{k}\right)_{k}$-adapted (real-valued) martingale differences under $\mathbb{P}_{\omega}$, and by Lemma A.9 combined with Remark 2.3 we can assume that

$$
\sum_{k \leq n s} E_{k-1}\left[\eta_{n k}^{2}\left(a_{1}, a_{2}\right)\right]+\sum_{n s<k \leq n t} E_{k-1}\left[\eta_{n k}^{2}\left(b_{1}, b_{2}\right)\right] \rightarrow_{n} \sigma_{\mathbf{u}, s, t}^{2}(\theta)
$$

$\mathbb{P}_{\omega}$-a.s. ${ }^{15}$ This verifies the first hypothesis in Theorem A.13 under $\mathbb{P}_{\omega}$ for all $\omega \in \Omega_{\theta}$ for the triangular array defining $U_{n}$.

It remains to prove that if $\omega \in \Omega_{\theta}$ then

$$
\sum_{k \leq n s} E_{0}\left[\eta_{n k}^{2}\left(a_{1}, a_{2}\right) I_{\left[\left|\eta_{n k}\left(a_{1}, a_{2}\right)\right|>\epsilon\right]}\right](\omega) \rightarrow 0 .
$$

\footnotetext{
${ }^{15}$ More precisely: redefine $\Omega_{\theta}$ above by intersecting it with the set $\Omega_{\theta}^{\prime}$ of elements $\omega$ for which the convergence in Lemma A.9 happens $\mathbb{P}_{\omega}$-a.s.
} 
This is, that for all $\omega \in \Omega_{\theta}$

$$
\sum_{k \leq n s} \eta_{n k}^{2}\left(a_{1}, a_{2}\right) I_{\left[\left|\eta_{n k}\left(a_{1}, a_{2}\right)\right|>\epsilon\right]} \rightarrow 0
$$

in $L_{\mathbb{P}_{\omega}}^{1}$.

To do so we depart from the Cauchy-Schwarz inequality to get that

$$
\eta_{n k}^{2}\left(x_{1}, x_{2}\right) \leq \frac{1}{n}\left(x_{1}^{2}+x_{2}^{2}\right) T^{k}\left|D_{0}(\theta)\right|^{2},
$$

so that the sum in (61) is bounded by

$$
\frac{1}{n} \sum_{k \leq n s} E_{0} T^{k}\left[\left(a_{1}^{2}+a_{2}^{2}\right)\left|D_{0}(\theta)\right|^{2} I_{\left[\left(a_{1}^{2}+a_{2}^{2}\right)\left|D_{0}(\theta)\right|^{2} \geq \epsilon^{2} n\right]}\right] .
$$

This obviously goes to zero when $a_{1}=a_{2}=0$. Otherwise it is the same as

$$
\left(a_{1}^{2}+a_{2}^{2}\right) \frac{1}{n} \sum_{k \leq n s} E_{0} T^{k}\left[\left|D_{0}(\theta)\right|^{2} I_{\left[\left|D_{0}(\theta)\right|^{2} \geq \epsilon^{2} n /\left(a_{1}^{2}+a_{2}^{2}\right)\right]}\right],
$$

which, again, goes to zero as $n \rightarrow \infty$ for every $\omega \in \Omega_{\theta}$.

Corollary 6.2. With the notation introduced in Theorem 5 (see also Remark 5.4), assume that the function

$$
(\theta, \omega) \mapsto D_{0}(\theta, \omega)
$$

is $\mathcal{B} \otimes \mathcal{F}$-measurable. Then there exists $\Omega_{0} \subset \Omega$ with $\mathbb{P} \Omega_{0}=1$ such that for all $\omega_{0} \in \Omega_{0}$ the asymptotic distribution of the $D[[0, \infty), \mathbb{C}]$-valued function $(\theta, \omega) \mapsto V_{n}(\theta, \omega)$ corresponds, un$\operatorname{der} \lambda \times \mathbb{P}_{\omega_{0}}$, to that of $\left(\theta, \omega^{\prime}\right) \mapsto B\left(\theta, \omega^{\prime}\right)$ under $\lambda \times \mathbb{P}^{\prime}$.

Proof. The function $(\theta, \omega) \mapsto V_{n}(\theta, \omega)$ is measurable with respect to $\mathcal{B} \otimes \mathcal{F}$ (see Section 4), and therefore by an application of Lemma A.11 in the Appendix and Proposition 2.2 above, the statement in Theorem 5 can be read in the following way: for any continuous and bounded function $f: D[[0, \infty), \mathbb{C}] \rightarrow \mathbb{R}$

$$
\lim _{n} E\left[f \circ V_{n} \mid \mathcal{B} \otimes \mathcal{F}_{0}\right](\theta, \omega)=E[f(B(\theta))]
$$

$\lambda \times \mathbb{P}$-a.s., where the expectation at the right denotes integration with respect to $\mathbb{P}^{\prime}$. This is an equality of $\mathcal{B} \times \mathcal{F}_{0}$ measurable functions, the $\mathcal{B}$-measurable function at the right being considered as constant in $\Omega$ for fixed $\theta$. An application of Theorem 34.2(v) in [6] gives that, for any given $\mathcal{B}_{0} \subset \mathcal{B}$

$$
\lim _{n} E\left[f \circ V_{n} \mid \mathcal{B}_{0} \otimes \mathcal{F}_{0}\right]=E\left[E[f(B(\theta))] \mid \mathcal{B}_{0} \otimes \mathcal{F}_{0}\right]
$$


$\lambda \times \mathbb{P}$-a.s. If $\mathcal{B}_{0}=\{\varnothing,[0,2 \pi)\}$ is the trivial sigma algebra then, as explained before, $\lambda_{\theta}:=\lambda$ for all $\theta \in[0,2 \pi)$ defines the regular measures corresponding to $E\left[\cdot \mid \mathcal{B}_{0}\right]$. It follows, under the light of Corollary A.12 in the Appendix and Proposition 2.2, that (62) is nothing but the statement of convergence $V_{n} \Rightarrow B$ under $\lambda \times \mathbb{P}_{\omega}$ for $\mathbb{P}$-a.e. $\omega$. This is the desired conclusion.

\section{Proof of Theorem 2}

We will present in this and the next section the proofs still pending from the results announced in Section 5. We continue working under the setting introduced in Section 3.

To begin with, let us prove the following "decomposition" lemma:

Lemma 7.1. For all $(n, r, \theta) \in \mathbb{N} \times \mathbb{N}^{*} \times[0,2 \pi)$ the following equality holds:

$$
\begin{aligned}
S_{n}(\theta)-E_{0} S_{n}(\theta)-M_{r, n}(\theta)= & -e^{i(n-1) \theta}\left(\sum_{k=1}^{r}\left(T^{n-1} E_{0} X_{k}-E_{0} T^{n-1} E_{0} X_{k}\right) e^{i k \theta}\right) \\
& +e^{i r \theta} \sum_{k=2}^{n-1}\left(T^{k} E_{-1} X_{r}-E_{0} T^{k} E_{-1} X_{r}\right) e^{i k \theta} \\
& -D_{r, 0}(\theta) .
\end{aligned}
$$

Proof. Fix $(n, r, \theta) \in \mathbb{N} \times \mathbb{N}^{*} \times[0,2 \pi)$. We depart from the following decomposition of $X_{0}$ (the array is intended to make visible the rearrangements):

$$
\begin{aligned}
X_{0}=E_{0} X_{0}= & \left(E_{0}-E_{-1}\right) X_{0}+E_{-1} X_{0} \\
& +\left(E_{0}-E_{-1}\right) X_{1} e^{i \theta}-\left(E_{0}-E_{-1}\right) X_{1} e^{i \theta} \\
& \vdots \\
& +\left(E_{0}-E_{-1}\right) X_{r} e^{i r \theta}-\left(E_{0}-E_{-1}\right) X_{r} e^{i r \theta} \\
= & \sum_{k=0}^{r}\left(\mathcal{P}_{0} X_{k}\right) e^{i k \theta}-\sum_{k=1}^{r}\left(E_{0} X_{k} e^{i k \theta}-E_{-1} X_{k-1} e^{i(k-1) \theta}\right) \\
& +E_{-1} X_{r} e^{i r \theta} .
\end{aligned}
$$

Now, using the equality

$$
\begin{aligned}
& \sum_{j=0}^{n-1} e^{i j \theta} T^{j} \sum_{k=1}^{r}\left(E_{0} X_{k} e^{i k \theta}-E_{-1} X_{k-1} e^{i(k-1) \theta}\right) \\
& =e^{i(n-1) \theta} T^{n-1} \sum_{k=1}^{r} E_{0} X_{k} e^{i k \theta}-\sum_{k=0}^{r-1} E_{-1} X_{k} e^{i k \theta}
\end{aligned}
$$


we get, from (64), that

$$
\begin{aligned}
S_{n}(\theta)= & M_{r, n}(\theta)-\left(e^{i(n-1) \theta} T^{n-1} \sum_{k=1}^{r} E_{0} X_{k} e^{i k \theta}-\sum_{k=0}^{r-1} E_{-1} X_{k} e^{i k \theta}\right) \\
& +\sum_{j=0}^{n-1} e^{i j \theta} T^{j} E_{-1} X_{r} e^{i r \theta}
\end{aligned}
$$

and that

$$
\begin{aligned}
E_{0} S_{n}(\theta)= & D_{r, 0}(\theta)-\left(E_{0} e^{i(n-1) \theta} T^{n-1} \sum_{k=1}^{r} E_{0} X_{k} e^{i k \theta}-\sum_{k=0}^{r-1} E_{-1} X_{k} e^{i k \theta}\right) \\
& +\sum_{j=0}^{n-1} e^{i j \theta} E_{0} T^{j} E_{-1} X_{r} e^{i r \theta} .
\end{aligned}
$$

Note that (63) follows from (65) and (66).

Let us denote by

$$
\begin{aligned}
& A_{r, n}=A_{r, n}(\theta, \omega):=\sum_{k=1}^{r}\left(T^{n-1} E_{0} X_{k}(\omega)-E_{0} T^{n-1} E_{0} X_{k}(\omega)\right) e^{i k \theta} \\
& B_{r, n}=B_{r, n}(\theta, \omega):=\sum_{k=0}^{n-1}\left(T^{k} E_{-1} X_{r}(\omega)-E_{0} T^{k} E_{-1} X_{r}(\omega)\right) e^{i k \theta}
\end{aligned}
$$

The following lemma is a key step for the proof of the validity of our martingale approximation.

Lemma 7.2. In the context of Lemma 7.1, and with the notation (67) and (68), there exists a constant $C>0$ such that, if $E_{0}$ is given by the regular version $E_{0} X(\omega)=E^{\omega} X\left(X \in L_{\mathbb{P}}^{1}\right)$ then

1. For all $(n, r, \theta) \in \mathbb{N} \times \mathbb{N}^{*} \times[0,2 \pi), \alpha \in \mathbb{R}$, and $\omega \in \Omega$

$$
E_{0}\left[\max _{k \leq n}\left|A_{r, k}(\theta, \cdot)\right|^{2}\right](\omega) \leq 4 \alpha^{2}+8 \sum_{j=0}^{n-1} E_{0} T^{j}\left|\left(E_{0} S_{r}(\theta)\right) I_{\left[\left|E_{0} S_{r}(\theta)\right|>\alpha\right]}\right|^{2}(\omega) .
$$

2. For all $\omega \in \Omega$

$$
\int_{0}^{2 \pi} E_{0}\left[\max _{k \leq n}\left|B_{r, k}(\theta, \cdot)\right|^{2}\right](\omega) d \lambda(\theta) \leq C \sum_{j=2}^{n-1} E_{0}\left|E_{j-1} X_{j+r}-E_{0} X_{j+r}\right|^{2}(\omega) .
$$


Proof. We will prove (69) using a truncation argument: let $U_{\alpha}$ be the (non-linear) operator given by $U_{\alpha} Y:=Y I_{|Y| \geq \alpha}$, and let us use the regular version of $E_{0}$, thus $E_{0} X(\omega)=E^{\omega} X\left(X \in L_{\mathbb{P}}^{1}\right)$. Then for all $\omega \in \Omega$

$$
\begin{aligned}
& \max _{k \leq n}\left|A_{r, k}(\theta, \cdot)\right|(\omega)=\max _{k \leq n}\left|\left(I d-E_{0}\right)\left(T^{k-1} E_{0} S_{r}(\theta)\right)\right|^{2}(\omega) \\
& \quad \leq 4 \alpha^{2}+2 \max _{k \leq n}\left|\left(I d-E_{0}\right) T^{k-1} U_{\alpha}\left(E_{0} S_{r}(\theta)\right)\right|^{2}(\omega) \\
& \quad \leq 4\left(\alpha^{2}+\sum_{j=0}^{n-1} T^{j}\left|U_{\alpha}\left(E_{0} S_{r}(\theta)\right)\right|^{2}(\omega)+\sum_{j=0}^{n-1} E_{0} T^{j}\left|U_{\alpha}\left(E_{0} S_{r}(\theta)\right)\right|^{2}\right)(\omega)
\end{aligned}
$$

where we used Jensen's inequality. This clearly implies (69).

Let us now prove (70): by Lemma A.7 there exists a constant $C$ such that

$$
\begin{aligned}
\int_{0}^{2 \pi} \max _{k \leq n}\left|B_{r, k}(\theta, z)\right|^{2} d \lambda(\theta) & \leq C \int_{0}^{2 \pi}\left|\sum_{j=2}^{n-1}\left(T^{j} E_{-1} X_{r}(z)-E_{0} T^{j} E_{-1} X_{r}(z)\right) e^{i j \theta}\right|^{2} d \lambda(\theta) \\
& =C \sum_{j=2}^{n-1}\left|E_{j-1} X_{j+r}(z)-E_{0} X_{j+r}(z)\right|^{2}
\end{aligned}
$$

The conclusion follows at once by integrating with respect to $\mathbb{P}_{\omega}$ over these inequalities and using Tonelli's theorem.

Proof of Theorem 2. Consider the notation introduced in Lemma 5.16. By an application of this lemma and Corollary 6.2, it suffices to prove that

$$
\tilde{E}_{0}\left[\frac{1}{n} \max _{1 \leq k \leq n}\left|S_{k}(\theta, \omega)-E_{0} S_{k}(\theta, \omega)-M_{r, k}(\theta, \omega)\right|^{2}\right]=o_{r}(1), \quad \mathbb{P} \text {-a.s. }
$$

Let us do so: by Lemma 7.1 and the definition of $\tilde{E}_{0}$, it is sufficient to prove that there exists $\Omega_{0}$ with $\mathbb{P} \Omega_{0}=1$ such that if for $(k, r, \theta) \in \mathbb{N} \times \mathbb{N}^{*} \times[0,2 \pi)$ we replace $Z_{r, k}(\theta, \omega):=A_{r, k}(\theta, \omega)$ or $Z_{r, k}(\theta, \omega):=B_{r, k}(\theta, \omega)$, then

$$
\lim _{r} \limsup _{n} \int_{0}^{2 \pi} E_{0}\left[\frac{1}{n} \max _{1 \leq k \leq n}\left|Z_{r, k}(\theta, \cdot)\right|^{2}\right](\omega) d \lambda(\theta)=0
$$

for all $\omega \in \Omega_{0}$.

Proof of (72) with $Z_{r, k}(\theta, \omega):=A_{r, k}(\theta, \omega)$ : if we fix the version of $E_{0}$ given by $E_{0} X(\omega)=$ $E^{\omega} X\left(X \in L_{\mathbb{P}}^{1}\right)$ then it is clear that for any $\omega \in \Omega$

$$
\left|E_{0} S_{r}(\theta) I_{\left[\left|E_{0} S_{r}(\theta)\right|>\alpha\right]}\right|(\omega) \leq\left|\left(\sum_{j=0}^{r-1} E_{0}\left|X_{j}\right|\right) I_{\left[\sum_{j=0}^{r-1} E_{0}\left|X_{j}\right|>\alpha\right]}\right|(\omega),
$$


and it follows by an application of (69), Birkhoff's Ergodic theorem, and Lemma A.6 in the Appendix (fixing first $\alpha>0$ so that the expectation of the random variable at the right hand side in (73) is less than any given $\eta>0$ ) that

$$
\lim _{n} E_{0}\left[\frac{1}{n} \max _{1 \leq k \leq n}\left|A_{r, k}(\theta, \cdot)\right|^{2}\right]=0 \quad \mathbb{P} \text {-a.s. }
$$

where the (probability one) set $\Omega_{0,1}$ of convergence does not depend on $\theta$ and, even more, the convergence is uniform in $\theta$ for any fixed $\omega \in \Omega_{0,1}$. It follows that for every $\omega \in \Omega_{0,1}$

$$
\begin{aligned}
& \underset{n}{\limsup } \int_{0}^{2 \pi} E_{0}\left[\frac{1}{n} \max _{1 \leq k \leq n}\left|A_{r, k}(\theta, \cdot)\right|^{2}\right](\omega) d \lambda(\theta) \\
& \leq \int_{0}^{2 \pi} \underset{n}{\lim \sup } E_{0}\left[\frac{1}{n} \max _{1 \leq k \leq n}\left|A_{r, k}(\theta, \cdot)\right|^{2}\right](\omega) d \lambda(\theta) \\
& \quad=0
\end{aligned}
$$

as desired.

Proof of (72) with $Z_{r, n}(\theta, \cdot):=B_{r, n}(\theta, \cdot)$ : again, fix the version of $E_{0}$ given by $E_{0} X(\omega)=$ $E^{\omega} X$. We depart from (70) and we note that, if for every $j \in \mathbb{Z}, X_{-\infty, j}:=X_{j}-E_{-\infty} X_{j}$ then

$$
\begin{aligned}
\sum_{k=2}^{n-1} E_{0}\left|\left(E_{k-1}-E_{0}\right) X_{k+r}\right|^{2} & =\sum_{k=2}^{n-1} E_{0}\left|\left(E_{k-1}-E_{0}\right) X_{-\infty, k+r}\right|^{2} \\
& =\sum_{k=2}^{n-1} E_{0} T^{k-1}\left|\left(E_{0}-E_{-k+1}\right) X_{-\infty, r+1}\right|^{2} \\
& =\sum_{k=1}^{n-2}\left(E_{0} T^{k}\left|E_{0} X_{-\infty, r+1}\right|^{2}-\left|E_{0} X_{-\infty, k+r+1}\right|^{2}\right) \\
& \leq \sum_{k=1}^{n-2} E_{0} T^{k}\left|E_{0} X_{-\infty, r+1}\right|^{2},
\end{aligned}
$$

$\mathbb{P}$-a.s. It follows from (70) and Lemma A.6 that

$$
\begin{aligned}
\underset{n}{\limsup } \int_{0}^{2 \pi} E_{0}\left[\frac{1}{n} \max _{1 \leq k \leq n}\left|B_{r, k}(\theta, \cdot)\right|^{2}\right](\omega) d \lambda(\theta) & \leq C\left\|E_{0} X_{-\infty, r+1}\right\|_{\mathbb{P}, 2}^{2} \\
& =C\left\|E_{-(r+1)} X_{-\infty, 0}\right\|_{\mathbb{P}, 2}^{2}
\end{aligned}
$$

$\mathbb{P}$-a.s. over a set $\Omega_{0,2, r}$ independent of $\theta$ and therefore, by the regularity condition (29) (see also (30))

$$
\lim _{r} \limsup _{n} \frac{1}{n} \int_{0}^{2 \pi} E_{0}\left[\max _{1 \leq k \leq n}\left|B_{r, k}(\theta, \cdot)\right|^{2}\right] d \lambda(\theta)=0
$$

for all $\omega \in \Omega_{0,2}:=\bigcap_{r \in \mathbb{N}} \Omega_{0,2, r}$. To conclude this part take $\Omega_{0}:=\Omega_{0,1} \cap \Omega_{0,2}$. 
We are left at this point with the statement on the convergence in distribution of (21) as $n \rightarrow \infty$ under the regularity condition (29).

To prove this claim, start by considering the inequalities $\left(S_{k}=S_{k}(\theta, \omega)\right.$ here, and similarly for $\left.M_{r, k}\right)$

$$
\begin{aligned}
\tilde{E}_{0} & \frac{1}{n} \max _{1 \leq k \leq n}\left|S_{k}-E_{0} S_{k}-M_{r, k}\right|^{2} \\
& \leq 2 \tilde{E}_{0} \frac{1}{n} \max _{1 \leq k \leq n}\left|S_{k}-M_{r, k}\right|^{2}+\frac{2}{n} \tilde{E}_{0} \max _{1 \leq k \leq n}\left|E_{0} S_{k}\right|^{2} \\
& \leq 4 \tilde{E}_{0} \frac{1}{n} \max _{1 \leq k \leq n}\left|S_{k}-E_{0} S_{k}-M_{r, k}\right|^{2}+\frac{6}{n} \tilde{E}_{0} \max _{1 \leq k \leq n}\left|E_{0} S_{k}\right|^{2}
\end{aligned}
$$

and notice that, by Lemma A.7 in the Appendix and $\mathcal{F}_{0}$-measurability, there exists a constant $C$ such that

$$
\frac{1}{n} \tilde{E}_{0} \max _{1 \leq k \leq n}\left|E_{0} S_{k}\right|^{2} \leq C \frac{1}{n} \sum_{k=0}^{n-1}\left|E_{0} X_{k}\right|^{2},
$$

$\mathbb{P}$-a.s. It follows from these observations and Corollary 5.14 that, under regularity, (71) is equivalent to

$$
\tilde{E}_{0}\left[\frac{1}{n} \max _{1 \leq k \leq n}\left|S_{k}(\theta, \omega)-M_{r, k}(\theta, \omega)\right|^{2}\right]=o_{r}(1), \quad \mathbb{P} \text {-a.s. }
$$

which gives the convergence of (21) under $\tilde{E}_{0}$ by the argument already given and an easy adaptation of Lemma 5.16.

\section{Proof of Theorems 3 and 4}

The first step towards the proof of Theorem 3 is the following martingale approximation lemma (here $S_{k}$ denotes non-rotated partial sums).

Lemma 8.1. In the context of Theorem 3

1. Assuming (38), there exists $D_{0} \in L_{\mathbb{P}}^{2}\left(\mathcal{F}_{0}\right) \ominus L_{\mathbb{P}}^{2}\left(\mathcal{F}_{-1}\right)$ such that, if $M_{n}$ is given by $M_{n}:=$ $\sum_{k=0}^{n-1} T^{k} D_{0}$, then

$$
\lim _{n} E_{0}\left[\frac{1}{n} \max _{1 \leq k \leq n}\left|S_{k}-E_{0} S_{k}-M_{k}\right|^{2}\right]=0, \quad \mathbb{P} \text {-a.s. }
$$

2. Assuming (40), there exists $D_{0} \in L_{\mathbb{P}}^{2}\left(\mathcal{F}_{0}\right) \ominus L_{\mathbb{P}}^{2}\left(\mathcal{F}_{-1}\right)$ such that, if $M_{n}$ is given by $M_{n}:=$ $\sum_{k=0}^{n-1} T^{k} D_{0}$

$$
\lim _{n} E_{0}\left[\frac{1}{n} \max _{1 \leq k \leq n}\left|S_{k}-M_{k}\right|^{2}\right]=0, \quad \mathbb{P} \text {-a.s. }
$$


Proof. The first statement is a part of Theorem 2.3 in [10]. The second statement is Theorem 2.7 in [8].

Remark 8.2. If $\left(a_{n}\right)_{n},\left(b_{n}\right)_{n}$ are sequences of complex numbers with $\left|b_{n}\right|$ increasing to $\infty$ and $a_{n} / b_{n} \rightarrow 0$, then

$$
\lim _{n} \max _{1 \leq k \leq n} \frac{\left|a_{k}\right|}{\left|b_{n}\right|}=0
$$

because

$$
0 \leq \lim \sup _{n} \max _{1 \leq k \leq n} \frac{\left|a_{k}\right|}{\left|b_{n}\right|} \leq \limsup \limsup _{n}\left(\frac{1}{\left|b_{n}\right|} \sum_{k=1}^{N-1}\left|a_{k}\right|+\sup _{k \geq N} \frac{\left|a_{k}\right|}{\left|b_{k}\right|}\right)=\lim _{N} \sup _{k \geq N} \frac{\left|a_{k}\right|}{\left|b_{k}\right|}=0 .
$$

If we apply this observation to the inequalities (76) with $\tilde{E}_{0}$ replaced by $E_{0}$ we see that, under the hypothesis

$$
\lim _{n} \frac{E_{0} S_{n}}{\sqrt{n}}=0 \quad \mathbb{P} \text {-a.s., }
$$

(78) and (79) are equivalent. We can actually verify (80) under (40) (see [8], Proposition 4.9) and therefore, under the (non-rotated) Maxwell and Woodroofe condition (40), (78) and (79) imply each other.

Remark 8.3. It is convenient, for the sake of clarity, to describe explicitly the martingale differences stated in Lemma 8.1: under (40), $D_{0}$ can be described as the $\mathbb{P}$-a.s. and in $L_{\mathbb{P}}^{2}$-convergent series

$$
D_{0}:=\sum_{n \geq 0} \sum_{k \geq n} \frac{\mathcal{P}_{0} X_{k}}{k+1}
$$

and under (38) $D_{0}$ is given by the $\mathbb{P}$-a.s. and in $L_{\mathbb{P}}^{2}$-convergent series

$$
D_{0}:=\sum_{k \geq 0} \mathcal{P}_{0} X_{k}
$$

Proof of Theorem 3. Start by considering the following observations: let $(\Omega, \mathcal{F}, \mathbb{P})$ be the underlying probability space (the domain of $X_{0}$ ), and for every measurable function $Y: \Omega \rightarrow \mathbb{C}$ let $\tilde{Y}$ be the extension to the product space $\tilde{Y}:[0,2 \pi) \times \Omega \rightarrow \mathbb{C}$ defined by

$$
\tilde{Y}(u, \omega):=e^{i u} Y(\omega) .
$$

For $\theta \in[0,2 \pi)$ fixed, define $\tilde{T}_{\theta}:[0,2 \pi) \times \Omega \rightarrow[0,2 \pi) \times \Omega$ by

$$
\tilde{T}_{\theta}(u, \omega):=((u+\theta) \bmod (2 \pi), T \omega)
$$

( $\tilde{T}_{\theta}$ is the product map between the rotation by an angle of $\theta$ and $T$ ). It is easy to see that $\tilde{T}_{\theta}$ is invertible, that it preserves the product measure $\lambda \times \mathbb{P}$, and that it is $\mathcal{B} \otimes \mathcal{F}$-bimeasurable. It is 
also easy to see that for every (measurable) $Y: \Omega \rightarrow \mathbb{C}$ and every $k \in \mathbb{Z}, \tilde{T}_{\theta}^{k} \tilde{Y}=\widehat{e^{i k \theta} T^{k}} Y$, and that $\|\tilde{Y}\|_{\lambda \times \mathbb{P}, 2}=\|Y\|_{\mathbb{P}, 2}$ if $Y \in L_{\mathbb{P}}^{2}$.

Denote, for every $k \in \mathbb{Z}, \tilde{\mathcal{F}}_{k}:=\mathcal{B} \otimes \mathcal{F}_{k}, \tilde{E}_{k}:=E\left[\cdot \mid \tilde{\mathcal{F}}_{k}\right]$ (conditional expectation with respect to $\lambda \times \mathbb{P}$ ), and $\tilde{\mathcal{P}}_{k}:=\tilde{E}_{k}-\tilde{E}_{k-1}$ (avoid confusion with the meaning of this notation in Lemma 5.16: here $\left.\mathcal{B}_{0}=\mathcal{B}\right)$. It is easy to see that $\left(\tilde{\mathcal{F}}_{k}\right)_{k \in \mathbb{Z}}$ is a $\tilde{T}_{\theta}$-filtration.

Recall also the notation introduced in Definition 5.1, and notice now that, if $\theta \in(0,2 \pi)$ and $Y \in L_{\mathbb{P}}^{1}$ is given then

$$
\left(1-e^{i \theta}\right) S_{n}(Y, T, \theta, \cdot)=T^{-1} Y+S_{n}\left(Y-T^{-1} Y, T, \theta, \cdot\right)-T^{n-1} Y e^{i n \theta} .
$$

Theorem 3 consists of two statements: the convergence of (20) under (37) or (39) and the convergence of (21) under (39). For reasons inherent to the logic of our arguments, we will prove first the convergence of (20) under (37), and then the convergence of (20) and (21) under (39).

Convergence of (20) under (37). First note that, without loss of generality, we can assume that $\left(X_{k}\right)_{k \in \mathbb{Z}}$ is regular (Definition 5.10). Indeed, with the notation introduced in Definition 5.1:

$$
\begin{aligned}
& S_{k}\left(X_{0}, T, \theta, \cdot\right)-E_{0} S_{k}\left(X_{0}, T, \theta, \cdot\right) \\
& \quad=S_{k}\left(X_{0}-E_{-\infty} X_{0}, T, \theta, \cdot\right)-E_{0} S_{k}\left(X_{0}-E_{-\infty} X_{0}, T, \theta, \cdot\right),
\end{aligned}
$$

and therefore we can study the desired asymptotics replacing the stationary process $\left(X_{k}\right)_{k \in \mathbb{Z}}$ by

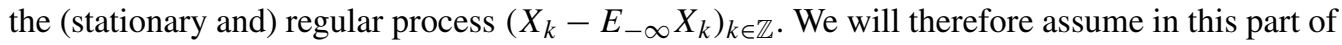
the proof that $\left(X_{k}\right)_{k \in \mathbb{Z}}$ is, indeed, regular.

Martingale approximations. For the sake of clarity, let us depart from the following observation: if we assume that

$$
\Delta_{0}(\theta):=\sum_{k \geq 0} \mathcal{P}_{0}\left(X_{k}-X_{k-1}\right) e^{i k \theta}
$$

converges $\mathbb{P}$-a.s. and in $L_{\mathbb{P}}^{2}$ then, necessarily

$$
D_{0}(\theta):=\sum_{k \geq 0} \mathcal{P}_{0} X_{k} e^{i k \theta}
$$

converges in $L_{\mathbb{P}}^{2}$ (under regularity). Even more,

$$
\left(1-e^{i \theta}\right) D_{0}(\theta)=\Delta_{0}(\theta)
$$

$\mathbb{P}$-a.s. To see this apply (83) and the definition of $\mathcal{P}_{0}$ to obtain, for every $r \in \mathbb{N}$, that

$$
\left(1-e^{i \theta}\right) \sum_{k=0}^{r} \mathcal{P}_{0} X_{k} e^{i k \theta}=\sum_{k=0}^{r} \mathcal{P}_{0}\left(X_{k}-X_{k-1}\right) e^{i k \theta}-\mathcal{P}_{0} X_{r} e^{i(r+1) \theta},
$$

and let $r \rightarrow \infty$ taking into account the regularity of $\left(X_{k}\right)_{k \in \mathbb{Z}}$. 
Now note that (37) implies that

$$
\sum_{k \geq 0}\left\|\mathcal{P}_{0}\left(X_{k}-X_{k-1}\right)\right\|_{\mathbb{P}, 2}=\sum_{k \geq 0}\left\|\tilde{\mathcal{P}}_{0}\left(\tilde{X}_{k}-\tilde{X}_{k-1}\right)\right\|_{\lambda \times \mathbb{P}, 2}<\infty .
$$

Taking $Y_{0}=X_{0}-X_{-1}$ and noticing that,

$$
\left\|\tilde{\mathcal{P}}_{0} \tilde{T}_{\theta}^{k} \tilde{Y}_{0}\right\|_{\lambda \times \mathbb{P}, 2}=\left\|\mathcal{P}_{0} T^{k} Y_{0}\right\|_{\mathbb{P}, 2}=\left\|\mathcal{P}_{0}\left(X_{k}-X_{k-1}\right)\right\|_{\mathbb{P}, 2}
$$

we see by Lemma 8.1 that we can find $\tilde{\Delta}_{0} \in L_{\lambda \times \mathbb{P}}^{2}\left(\tilde{\mathcal{F}}_{0}\right) \ominus L_{\lambda \times \mathbb{P}}^{2}\left(\tilde{\mathcal{F}}_{-1}\right)$ such that

$$
\begin{aligned}
& \lim _{n} \frac{1}{n} \tilde{E}_{0} \max _{1 \leq k \leq n}\left|S_{k}\left(\tilde{X}_{0}-\tilde{X}_{-1}, \tilde{T}_{\theta}, 0, \cdot\right)-E_{0} S_{k}\left(\tilde{X}_{0}-\tilde{X}_{-1}, \tilde{T}_{\theta}, 0, \cdot\right)-S_{k}\left(\tilde{\Delta}_{0}, \tilde{T}_{\theta}, 0, \cdot\right)\right|^{2} \\
& \quad=0
\end{aligned}
$$

$\lambda \times \mathbb{P}$-a.s. Even more, using Remark 8.3 we see that

$$
\tilde{\Delta}_{0}(u, \omega)=e^{i u} \sum_{k \geq 0} \mathcal{P}_{0}\left(X_{k}-X_{k-1}\right)(\omega) e^{i k \theta} .
$$

This gives that if $\Delta_{0}(\theta, \omega):=e^{-i u} \tilde{\Delta}_{0}(u, \omega)$ then

$$
\begin{aligned}
& \lim _{n} \frac{1}{n} E_{0} \max _{1 \leq k \leq n}\left|S_{k}\left(X_{0}-X_{-1}, T, \theta, \cdot\right)-E_{0} S_{k}\left(X_{0}-X_{-1}, T, \theta, \cdot\right)-S_{k}\left(\Delta_{0}(\theta), T, \theta, \cdot\right)\right|^{2} \\
& \quad=0
\end{aligned}
$$

$\mathbb{P}$-a.s., which is the same, by (83), (86) and the convergence $E_{0}\left|\left(I d-E_{0}\right) X_{n}\right|^{2}=o(n), \mathbb{P}$-a.s. (use for instance the pointwise ergodic theorem for $E_{0} T$, see also the first part of Remark 8.2), as

$$
\begin{aligned}
& \left|1-e^{i \theta}\right|^{2} \lim _{n} \frac{1}{n} E_{0} \max _{1 \leq k \leq n}\left|\left(S_{k}\left(X_{0}, T, \theta, \cdot\right)-E_{0} S_{k}\left(X_{0}, T, \theta, \cdot\right)-S_{k}\left(D_{0}(\theta), T, \theta, \cdot\right)\right)\right|^{2} \\
& \quad=0
\end{aligned}
$$

$\mathbb{P}$-a.s., where $D_{0}(\theta)$ is given by (85). The result follows at once from Lemma 5.6 assuming that $e^{2 i \theta} \notin \operatorname{Spec}_{p}(T)$ (in particular $e^{i \theta} \neq 1$ ).

Convergence of (20) and (21) under (39). With the notation already introduced, note that

$$
\sum_{k \geq 1} \frac{\left\|E_{0} S_{k}\left(X_{0}, T, \theta, \cdot\right)\right\|_{\mathbb{P}, 2}}{k^{3 / 2}}=\sum_{k \geq 1} \frac{\left\|\tilde{E}_{0} S_{k}\left(\tilde{X}_{0}, \tilde{T}_{\theta}, 0, \cdot\right)\right\|_{\lambda \times \mathbb{P}, 2}}{k^{3 / 2}}<\infty
$$

and it follows, by applying Lemma 8.1 again, that if $\tilde{D}_{0}$ is defined by

$$
\tilde{D}_{0}:=\sum_{n \geq 0} \sum_{k \geq n} \frac{\tilde{\mathcal{P}}_{0} \tilde{T}_{\theta}^{k} \tilde{X}_{0}}{k+1}
$$


then

$$
\lim _{n} \frac{1}{n} \tilde{E}_{0} \max _{1 \leq k \leq n}\left|S_{k}\left(\tilde{X}_{0}, \tilde{T}_{\theta}, 0, \cdot\right)-S_{k}\left(\tilde{D}_{0}, \tilde{T}_{\theta}, 0, \cdot\right)\right|^{2}=0
$$

$\lambda \times \mathbb{P}$-a.s. By arguments similar to those preceding (91), this is the same as saying that if

$$
D_{0}(\theta)=\sum_{n \geq 0} \sum_{k \geq n} \frac{\mathcal{P}_{0} X_{k} e^{i k \theta}}{k+1}
$$

then

$$
\lim _{n} \frac{1}{n} E_{0} \max _{1 \leq k \leq n}\left|S_{k}\left(X_{0}, T, \theta, \cdot\right)-S_{k}\left(D_{0}(\theta), T, \theta, \cdot\right)\right|^{2}=0,
$$

$\mathbb{P}$-a.s. This implies the desired conclusion by (an easy adaptation of) Lemma 5.6. The convergence of (20) follows by a similar argument after applying Remark 8.2 to (94).

Proof of Theorem 4. We start from the following observation: if $\left(a_{k}\right)_{k \in \mathbb{N}}$ is a sequence of nonnegative numbers, $\left(b_{k}\right)_{k \in \mathbb{N}}$ is a sequence of (strictly) positive numbers, and $n \in \mathbb{N}^{*}$ is given, an application of Hölder's inequality gives that

$$
\sum_{k=1}^{n} \frac{a_{k}}{k^{3 / 2}} \leq\left(\sum_{k=1}^{n} \frac{1}{k b_{k}}\right)^{1 / 2}\left(\sum_{k=1}^{n} \frac{a_{k}^{2} b_{k}}{k^{2}}\right)^{1 / 2}
$$

and therefore the convergence of $\sum_{k} a_{k} / k^{3 / 2}(k \geq 1)$ is equivalent to the existence of a positive sequence $\left(b_{k}\right)_{k \in \mathbb{N}}$ such that both $\sum_{k} 1 /\left(k b_{k}\right)$ and $\sum_{k} a_{k}^{2} b_{k} / k^{2}$ are convergent (for the necessity consider $b_{k}:=k^{1 / 2} / a_{k}$ if $a_{k}>0$ and $b_{k}=k$ otherwise).

This observation applied to $a_{k}:=\left\|E_{0} S_{k}(\theta)\right\|_{\mathbb{P}, 2}$ (for $\theta \in[0,2 \pi$ ) fixed) gives that a sufficient condition for the fulfillment of (39) is the existence of $\beta>1$ such that

$$
\sum_{k \in \mathbb{N}^{*}}(\log k)^{\beta} \frac{E\left|E_{0} S_{k}(\theta)\right|^{2}}{k^{2}}<\infty
$$

In order to provide a condition giving rise to the fulfillment of (97) for $\lambda$-a.e. $\theta$, we fix $n \in \mathbb{N}^{*}$ and start by noticing that, by orthogonality and Fubini's theorem

$$
\begin{aligned}
\int \sum_{k=1}^{n}(\log k)^{\beta} \frac{\left\|E_{0} S_{k}(\theta)\right\|_{\mathbb{P}, 2}^{2}}{k^{2}} d \lambda(\theta) & =\sum_{k=1}^{n} \sum_{j=0}^{k-1} \frac{(\log k)^{\beta}}{k^{2}}\left\|E_{0} X_{j}\right\|_{\mathbb{P}, 2}^{2} \\
& =\sum_{j=0}^{n-1}\left\|E_{0} X_{j}\right\|_{\mathbb{P}, 2}^{2} \sum_{k=j+1}^{n} \frac{(\log k)^{\beta}}{k^{2}},
\end{aligned}
$$


and that one can show (using for instance the integral test and integration by parts) that for every $\beta>1$, there exists $C(\beta)>0$ such that

$$
\sum_{k=j+1}^{n} \frac{(\log k)^{\beta}}{k^{2}} \leq C(\beta) \frac{(\log (j))^{\beta}}{j},
$$

for every $1 \leq j \leq n$ given. This, together with Tonelli's theorem, shows that

$$
\int \sum_{k=1}^{\infty}(\log k)^{\beta} \frac{\left\|E_{0} S_{k}(\theta)\right\|_{\mathbb{P}, 2}^{2}}{k^{2}} d \lambda(\theta) \leq C(\beta) \sum_{k=1}^{\infty}(\log k)^{\beta} \frac{\left\|E_{0} X_{k}\right\|_{\mathbb{P}, 2}^{2}}{k},
$$

$\mathbb{P}$-a.s. With this Theorem 4 follows from (42) and Theorem 3.

\section{Appendix}

In this section, we provide some results used along the proofs of the statements previously given. Some of these results belong to the existing literature and are included here for the sake of clarity, the rest of them are either not very visible in the mainstream literature or new, and we include them in this section due to their general scope.

We start by giving a further equivalence to the Portmanteau theorem, valid in the case of separable metric spaces, and whose relevance for our arguments lies in the fact that it reduces the "integral testing" for convergence in distribution to a countable set of functions.

To introduce this result, first remember the notion of a Urysohn function: given two closed, disjoint sets $F_{0}, F_{1}$ in a perfectly normal topological space (for instance, any metric space) $\mathcal{T}$,

$$
U=U\left(F_{0}, F_{1}\right): \mathcal{T} \rightarrow[0,1]
$$

is called a Urysohn function if it is continuous, $U^{-1}\{0\}=F_{0}$ and $U^{-1}\{1\}=F_{1}$.

Let us call a collection $\left\{F_{j}\right\}_{j \in J}$ of closed sets in $\mathcal{T}$ a co-base if $\left\{\mathcal{T} \backslash F_{j}\right\}_{j \in J}$ is a base of $\mathcal{T}$. We will also use the following notation: if $S$ is a metric space with distance function $d$, then for any given $x \in S$ and $A \subset S$ (not necessarily in the topology of $S$ ) we define the distance from $x$ to $A$ by

$$
d(x, A):=\inf _{a \in A} d(x, a),
$$

and we define the $\epsilon$-neighborhood of $A, A^{\epsilon}$, as the (open) set

$$
A^{\epsilon}:=\{x \in S: d(x, A)<\epsilon\} .
$$

Lemma A.1. Let $S$ be a separable metric space. Denote by $\mathbf{C}^{b}(S)$ the space of bounded, continuous real-valued functions on $S$. Let $\left\{F_{n}\right\}_{n \in \mathbb{N}}$ be a co-base of $S$ which is also a $\pi$-system, and let $X_{n}, X(n \in \mathbb{N})$ be random elements of $S$ (not necessarily defined on the same probability space). Then the following two statements are equivalent 
1. For every $f \in \mathbf{C}^{b}(S)$,

$$
\lim _{n} E f\left(X_{n}\right)=E f(X) .
$$

2. For every $k \in \mathbb{N}$, every rational $\epsilon>0$, and some Urysohn function $U_{k, \epsilon}=U\left(S \backslash F_{k}^{\epsilon}, F_{k}\right)$

$$
\lim _{n} E U_{k, \epsilon}\left(X_{n}\right)=E U_{k, \epsilon}(X)
$$

Proof. Denote by $P_{n}$ the law of $X_{n}$ and by $P$ the law of $X$. Since 1 . clearly implies 2 . it suffices to see, by the Portmanteau theorem ([7], Theorem 2.1), that if 2 . is true then for any given closed set $F$

$$
\limsup _{n} P_{n} F \leq P F .
$$

If for some $k, F=F_{k}$, this is a consequence of the inequalities

$$
I_{F} \leq U_{k, \epsilon} \leq I_{F} \epsilon
$$

the hypothesis in 2. and the continuity from above of finite measures.

If $F$ is an arbitrary closed set, say $F=\bigcap_{j \in J} F_{j}$ for some $J \subset \mathbb{N}$, and if we define for all $k \in \mathbb{N}, J_{k}:=J \cap[0, k]$ and $A_{k}:=\bigcap_{j \in J_{k}} F_{j}$ then, since $A_{k} \in\left\{F_{n}\right\}_{n}$,

$$
\limsup _{n} P_{n} F \leq \limsup _{n} P_{n} A_{k} \leq P A_{k}
$$

for all $k$. By letting $k \rightarrow \infty$ we get the desired conclusion.

We remark that the Portmanteau theorem can be extended to the context of abstract perfectly normal spaces if one interprets convergence in distribution as the fulfillment of the hypothesis 1 . of Lemma A.1. This can be seen by following the arguments in [7] and using the fact that every closed set is a $G_{\delta}$ set. In this context, Lemma A. 1 corresponds to the second-countable case.

Our next result, Theorem A.2, is an improvement due to Dehling, Durieu and Volny, of Theorem 3.1 in [7] for the case in which the target (state) space is a complete and separable metric space. As in the previous pages, “ $\Rightarrow$ ” denotes convergence in distribution.

Theorem A.2. Let $(S, d)$ be a complete and separable metric space. Assume that for all $r, n \geq 0$, $X_{(r, n)}$ and $X_{n}$ are random elements of $S$ defined on the same probability space $(\Omega, \mathcal{F}, \mu)$, and that $X_{(r, n)} \Rightarrow_{n} Z_{r}$. Then the hypothesis

$$
\lim _{r} \limsup _{n} \mu\left[d\left(X_{(r, n)}, X_{n}\right) \geq \epsilon\right]=0, \quad \text { for all } \epsilon>0
$$

implies the existence of a random element $X$ of $S$ such that $Z_{r} \Rightarrow_{r} X$ and $X_{n} \Rightarrow_{n} X$.

Proof. This is Theorem 2 in [12]. 
Corollary A.3. In the context of Theorem A.2 denote, for any given $q>0$,

$$
\|Z\|_{\mu, q}:=\left(\int_{\Omega}|Z|^{q} d \mu(\omega)\right)^{1 / q} .
$$

If for some $q>0$

$$
\lim _{r} \limsup _{n}\left\|d\left(X_{(r, n)}, X_{n}\right)\right\|_{\mu, q}=0
$$

and $X_{(r, n)} \Rightarrow_{n} Z_{r}$, then there exists a random element $X$ such that $X_{n} \Rightarrow_{n} X$.

Proof. Apply Markov's inequality to verify the hypothesis of Theorem A.2.

The following lemma is used without a proof along the references consulted by the author, thus a demonstration is given. This result allows us to pass from the study of stationary martingales to martingales under the conditional regular measures (see Section 3).

Lemma A.4. With the notation and definitions given in Section 3, and denoting further by $E_{k}^{\omega}$ the conditional expectation with respect to $\mathcal{F}_{k}$ and $\mathbb{P}_{\omega}$, the following property holds: for every $k \in \mathbb{N}$, every $\mathbb{P}$-integrable $Y$, and every fixed version of $E_{k} Y$ (also denoted by $E_{k} Y$ ):

$$
E_{k}^{\omega} Y=E_{k} Y
$$

$\mathbb{P}_{\omega}$-a.s. for $\mathbb{P}$-a.e. $\omega$. In particular, if $\left(M_{n}\right)_{n}$ is an $\left(\mathcal{F}_{n}\right)_{n}$-adapted martingale in $L_{\mathbb{P}}^{p}(p \geq 1)$, then $\left(M_{n}\right)_{n \geq 0}$ is an $\left(\mathcal{F}_{n}\right)_{n}$-adapted martingale in $L_{\mathbb{P}_{\omega}}^{p}$ for $\mathbb{P}$-a.e. $\omega$.

Proof. Fix a version of $Y \in L_{\mathbb{P}}^{1}$. We will prove that for any $\left(\mathcal{F}_{k}\right.$-measurable) version of $E_{k} Y$, there exists a set $\Omega_{Y} \subset \Omega$ with $\mathbb{P} \Omega_{Y}=1$ such that the following holds: for every $\omega \in \Omega_{Y}$ and every $A \in \mathcal{F}_{k}$

$$
\int_{A} Y(z) d \mathbb{P}_{\omega}(z)=\int_{A} E_{k} Y(z) d \mathbb{P}_{\omega}(z),
$$

this clearly implies the first conclusion.

Fix a $\left(\mathcal{F}_{k}\right.$-measurable) version of $E_{k} Y$ and notice that for $A$ fixed, a set $\Omega_{Y, A}$ of probability one such that (A.3) holds for all $\omega \in \Omega_{Y, A}$ exists by the property defining the family $\left\{\mathbb{P}_{\omega}\right\}_{\omega \in \Omega}$ and because

$$
E_{0}\left[Y I_{A}\right]=E_{0}\left[\left(E_{k} Y\right) I_{A}\right]
$$

$\mathbb{P}$-a.s. Without loss of generality $\Omega_{Y, A} \subset\left\{\omega \in \Omega:|Y|+\left|E_{k} Y\right| \in L_{\mathbb{P}_{\omega}}^{1}\right\}$ (the last set has $\mathbb{P}$-measure one because $E|Z|=E E_{0}|Z|$ for every $\left.Z \in L_{\mathbb{P}}^{1}\right)$.

Now proceed as follows: let $\left\{A_{n}\right\}_{n \in \mathbb{N}} \subset \mathcal{F}_{k}$ be a countable family generating $\mathcal{F}_{k}$ which is also a $\pi$-system and includes $\Omega$ (such a family exists because $\mathcal{F}_{0}$ is assumed countably generated), let $\Omega_{Y}:=\bigcap_{n \geq 1} \Omega_{Y, A_{n}}$, and let $\mathcal{G}_{k} \subset \mathcal{F}_{k}$ be the family of sets $A \in \mathcal{F}_{k}$ such that (A.3) holds for all $\omega \in \Omega_{Y}$. It is easy to see that $\mathcal{G}_{k}$ is a $\lambda$-system and therefore, since it includes $\left\{A_{n}\right\}_{n \in \mathbb{N}}$, the 
$\pi-\lambda$ theorem implies that $\mathcal{G}_{k}=\mathcal{F}_{k}$. Note that $\mathbb{P} \Omega_{Y}=1$, and that for all $\omega \in \Omega_{Y}$, (A.3) holds for all $A \in \mathcal{F}_{k}$.

This gives the proof of the first conclusion. The second conclusion (the one about martingales) follows easily from this, together with the fact that $E|X|^{p}=E E_{0}|X|^{p}$ and therefore if $E|X|^{p}<$ $\infty$ then $E^{\omega}|X|^{p}<\infty$ for $\mathbb{P}$-a.e. $\omega$.

Recall the following (Doob's) maximal inequality ([22], p. 53): if $p>1$ is given and $\left(M_{k}\right)_{k}$ is a nonnegative submartingale in $L_{\mu}^{p}$ then

$$
\left\|M_{n}\right\|_{p, \mu} \leq\left\|\max _{0 \leq k \leq n} M_{k}\right\|_{p, \mu} \leq \frac{p}{p-1}\left\|M_{n}\right\|_{p, \mu} .
$$

A combination of Doob's maximal inequality (A.4) with Lemma A.4 gives the following lemma.

Lemma A.5. With the notation of Section 3, if $\left(M_{k}\right)_{k}$ is an $\left(\mathcal{F}_{k}\right)_{k}$-adapted complex martingale in $L_{\mathbb{P}}^{2}$ then

$$
E_{0}\left[\max _{0 \leq k \leq n}\left|M_{k}\right|\right]^{2} \leq 4 E_{0}\left|M_{n}\right|^{2}, \quad \mathbb{P} \text {-a.s. }
$$

We also need in this paper the following ergodic theorem, which was demonstrated at the beginning of the proof of Propostion 5.12.

Lemma A.6. With the notation introduced in Section 3, and assuming $T$ is ergodic, for every $Y \in L_{\mathbb{P}}^{1}$,

$$
\lim _{n} \frac{1}{n} \sum_{k=0}^{n-1} E_{0} T^{k} Y=E Y
$$

$\mathbb{P}$-a.s. and in $L_{\mathbb{P}}^{1}$.

Proof. See the proof of Proposition 5.12. ${ }^{16}$

The following lemma is a classical tool in Harmonic Analysis, we give here a concrete version sufficient for our purposes.

Lemma A.7. There exists a constant $C$ with the following property: for any given $f \in L_{\lambda}^{2}$ with Fourier expansion

$$
S_{f}(\theta)=\sum_{k \geq 0} a_{k} e^{i k \theta},
$$

\footnotetext{
${ }^{16}$ Or see Lemma 7.1 in [11] for a slightly different version of this result (which also inspired it).
} 
and denoting by $S_{f, n}(\theta):=\sum_{k=0}^{n-1} a_{k} e^{i k \theta}$ the $n$th Fourier partial Fourier sum of $f$ :

$$
\int_{[0,2 \pi)} \sup _{n}\left|S_{f, n}(\theta)\right|^{2} d \lambda(\theta) \leq C \int_{[0,2 \pi)}|f(\theta)|^{2} d \lambda(\theta) .
$$

Proof. [17].

The next lemma is useful to compute the finite-dimensional asymptotic distributions that identify our asymptotic (quenched) limits.

Lemma A.8. Let $(\Omega, \mathcal{F}, \mathbb{P})$ be a probability space, let $T: \Omega \rightarrow \Omega$ be a measure preserving transformation and let $\theta \in \mathbb{R}$. If the only integrable (complex-valued) function $Y$ satisfying $T Y=$ $e^{-i \theta} Y$ is $Y=0$ (i.e., if $e^{-i \theta} \notin \operatorname{Spec}_{p}(T)$ ), then for every $X \in L_{\mathbb{P}}^{1}(\Omega, \mathbb{C})$

$$
\lim _{n} \frac{1}{n} \sum_{k=0}^{n-1} T^{k} X e^{i k \theta}=0, \quad \mathbb{P} \text {-a.s. and in } L_{\mathbb{P}}^{1} \text {. }
$$

Proof. [9], p. $20 .^{17}$

The following lemma is a corollary of the previous one. Its proof is basically the same as that of the equality (16) in [9]. ${ }^{18}$

Lemma A.9. With the notations and definitions given in Section 3 and Section 5, assume that $\theta \in[0,2 \pi)$ is such that $e^{-2 i \theta} \notin \operatorname{Spec}_{p}(T)$ and let $Y_{0} \in L_{\mathbb{P}}^{2}$ be given. Then for every $\mathbf{x}=\left(x_{1}, x_{2}\right)$

$$
\lim _{n} \frac{1}{n} \sum_{k=0}^{n-1} E_{k-1}\left(\mathbf{x} \cdot\left(T^{k} Y_{0} e^{i k \theta}\right)\right)^{2}=\frac{\left(x_{1}^{2}+x_{2}^{2}\right)}{2} E\left|Y_{0}\right|^{2}, \quad \text { P-a.s. and in } L_{\mathbb{P}}^{1},
$$

where the (probability one) set of pointwise convergence does not depend on $x_{1}, x_{2}$.

Proof. Notice that

$$
E_{k-1}\left(\mathbf{x} \cdot\left(T^{k} Y_{0} e^{i k \theta}\right)\right)^{2}=T^{k} E_{-1}\left(\mathbf{x} \cdot\left(Y_{0} e^{i k \theta}\right)\right)^{2}
$$

and adapt the argument leading to (16) in [9] (alternatively, see the proof of Lemma 5 in [4]).

\footnotetext{
${ }^{17}$ Actually, as proved for instance in [4], Theorem 3.2, if $X \in L_{\mathbb{P}}^{p}$ for some $p \geq 1$ and $\theta$ is arbitrary, the random variables at the left in (A.7) converge $\mathbb{P}$-a.s. and in $L_{\mathbb{P}}^{p}$, as $n \rightarrow \infty$, to the orthogonal projection of $X$ on the (in the ergodic case, at most one-dimensional) space of functions $Y \in L_{\mathbb{P}}^{p}$ with $T Y=e^{-i \theta} Y$.

${ }^{18}$ There is a typo in [9]: according to the notation there (avoid confusion with our notation) the correct statement is the following: if $e^{-2 i t}$ is not an eigenvalue of $\theta,(16)$ is valid $\mathbb{P}$-a.s. (not $\tilde{\mathbb{P}}$-a.s.) for every fixed $u$. An analysis of the proof shows that the convergence is valid also in the $L_{\mathbb{P}}^{1}$-sense, which is not explicitly stated there.
} 
Remark A.10. The set of probability one in Lemma A.9 can be described as the set where the pointwise convergences

$$
\begin{aligned}
\frac{1}{n} \sum_{k=0}^{n-1} T^{k} E_{-1}\left[\left(\operatorname{Re}\left(Y_{0}\right)\right)^{2}\right] e^{i 2 k \theta} \rightarrow_{n} 0, & \frac{1}{n} \sum_{k=0}^{n-1} T^{k} E_{-1}\left[\left(\operatorname{Im}\left(Y_{0}\right)\right)^{2}\right] e^{i 2 k \theta} \rightarrow_{n} 0, \\
\frac{1}{n} \sum_{k=0}^{n-1} T^{k} E_{-1}\left[\operatorname{Re}\left(Y_{0}\right) \operatorname{Im}\left(Y_{0}\right)\right] e^{i 2 k \theta} \rightarrow_{n} 0, & \frac{1}{n} \sum_{k=0}^{n-1} T^{k} E_{-1}\left|Y_{0}\right|^{2} \rightarrow_{n} E\left|Y_{0}\right|^{2}
\end{aligned}
$$

hold (for fixed versions of the functions involved). The details are left to the reader (alternatively, see the proof of Lemma 5 in [4]).

The following lemma has a very classical flavor but it is not visible in the literature. We use it in Section 3 to understand systematically the nature of the quenched results obtained in the paper, and for the proof of Corollary 6.2 .

Lemma A.11. Let $(\Theta, \mathcal{B}, \lambda)$ and $(\Omega, \mathcal{F}, \mathbb{P})$ be probability spaces and let $\mathcal{F}_{0} \subset \mathcal{F}$ be a sigma algebra such that $E\left[\cdot \mid \mathcal{F}_{0}\right]$ admits a regular version in the sense explained in Section 3: there exists a family of probability measures $\left\{\mathbb{P}_{\omega}\right\}_{\omega \in \Omega}$ such that for every (version of) $Y \in L_{\mathbb{P}}^{1}$,

$$
\omega \mapsto \int_{\Omega} Y(z) d \mathbb{P}_{\omega}(z)
$$

defines an $\mathcal{F}_{0}$-measurable version of $E\left[Y \mid \mathcal{F}_{0}\right]$. Define, for any (version of) $f \in L_{\lambda \times \mathbb{P}}^{1}$,

$$
\tilde{f}(\theta, \omega):=\int_{\Omega} f(\theta, z) d \mathbb{P}_{\omega}(z)
$$

provided that the integral exists, and zero otherwise. Then $\tilde{f}$ is a version of $E\left[f \mid \mathcal{B} \otimes \mathcal{F}_{0}\right]$.

Proof. Note that, by Fubini's theorem, $f(\theta, \cdot) \in L_{\mathbb{P}}^{1}$ for $\lambda$-a.e. $\theta$, and that for such $\theta, \tilde{f}(\theta, \cdot)$ is a version of $E_{0}[f(\theta, \cdot)]$. If we succeed proving that $\tilde{f}$ is $\mathcal{B} \otimes \mathcal{F}_{0}$-measurable it follows by Fubini's theorem again that given any rectangular set $E=A \times B \in \mathcal{B} \otimes \mathcal{F}_{0}$

$$
\int_{E} f(\theta, \omega) d(\lambda \times \mathbb{P})=\int_{A} \int_{B} f(\theta, \omega) d \mathbb{P}(\omega) d \lambda(\theta)=\int_{A} \int_{B} \tilde{f}(\theta, \omega) d \mathbb{P}(\omega) d \lambda(\theta),
$$

so that, by an application of the $\pi-\lambda$ theorem similar to the one at the end of this proof,

$$
\int_{E} f(\theta, \omega) d(\lambda \times \mathbb{P})=\int_{E} \tilde{f}(\theta, \omega) d(\lambda \times \mathbb{P})
$$

for all $E \in \mathcal{B} \otimes \mathcal{F}_{0}$. Thus it suffices to prove the $\mathcal{B} \otimes \mathcal{F}_{0}$-measurability of $\tilde{f}$. 
Note that if $f^{+}:=f I_{[f \geq 0]}$ and $f^{-}:=-f I_{[f<0]}$ are, respectively, the nonnegative and negative parts of a real-valued $f \in L_{\lambda \times \mathbb{P}}^{1}$, we can recover the definition of $\tilde{f}$ via the formula

$$
\tilde{f}=\left(\tilde{f^{+}}-\tilde{f^{-}}\right) I_{[|\widetilde{f}|>0]}
$$

(here $\widetilde{f} \mid:=\tilde{g}$ with $g:=|f|$ ), and we can create a (measurable) formula for an arbitrary $f \in$ $L_{\lambda \times \mathbb{P}}^{1}$ by applying this to its real and imaginary parts.

Thus it suffices to assume that $f$ is nonnegative. We will do so for the rest of the proof.

It is well known that every nonnegative function $f$ can be approximated by simple functions $f_{n}$ with $f_{n}$ increasing to $f$ ([6], Theorem 13.5, p. 185). Then, by the monotone convergence theorem,

$$
\tilde{f}=\lim _{n}\left(\tilde{f}_{n} I_{A_{f}}\right)
$$

where

$$
A_{f}:=\bigcup_{n \in \mathbb{N}} \bigcap_{k \in \mathbb{N}}\left[\tilde{f}_{k} \leq n\right] .
$$

Thus, it suffices to see that for each simple function $f, \tilde{f}$ is $\mathcal{B} \otimes \mathcal{F}_{0}$-measurable, and therefore it suffices (by linearity) to prove this if $f=I_{E}$ for any $E \in \mathcal{B} \otimes \mathcal{F}$.

Let us do it: if $E=A \times B$ is a rectangular set, then

$$
\tilde{I}_{E}(\theta, \omega)=I_{A}(\theta) \mathbb{P}_{\omega}(B),
$$

which is clearly $\mathcal{B} \otimes \mathcal{F}_{0}$ measurable. It follows that $\tilde{I}_{E}$ is $\mathcal{B} \otimes \mathcal{F}_{0}$-measurable if $E$ is any finite union of disjoint rectangles in $\mathcal{B} \otimes \mathcal{F}$.

Now consider the family $\tilde{\mathcal{F}}^{\prime}$ of sets $E \in \mathcal{B} \otimes \mathcal{F}$ such that $\tilde{I}_{E}$ is $\mathcal{B} \otimes \mathcal{F}_{0}$-measurable. Since for any family $\left\{E_{n}\right\}_{n} \subset \tilde{\mathcal{F}}^{\prime}$ of mutually disjoint sets

$$
\tilde{I}_{\cup_{n}} E_{n}=\sum_{n} \tilde{I}_{E_{n}}
$$

(apply the monotone convergence theorem) and $\Theta \times \Omega$ is an element of $\tilde{\mathcal{F}}^{\prime}, \tilde{\mathcal{F}}^{\prime}$ is a $\lambda$-system. Since $\tilde{\mathcal{F}}^{\prime}$ includes the finite unions of disjoint rectangles it follows, by the $\pi-\lambda$ theorem, that $\tilde{\mathcal{F}}^{\prime}=\mathcal{B} \otimes \mathcal{F}$.

Corollary A.12. Under the conditions of Lemma A.11, if $\mathcal{B}_{0} \subset \mathcal{B}$ is a sigma algebra such that $E\left[\cdot \mid \mathcal{B}_{0}\right]$ is regular with regular measures $\left\{\lambda_{\theta}\right\}_{\theta \in \Theta}$, so that

$$
\theta \mapsto \int_{\Theta} g(z) d \lambda_{\theta}(z)
$$

is $\mathcal{B}_{0}$-measurable and defines a version of $E\left[g \mid \mathcal{B}_{0}\right]$ for any $g \in L_{\lambda}^{1}$, then $\left\{\lambda_{\theta} \times \mathbb{P}_{\omega}\right\}_{(\theta, \omega) \in \Theta \times \Omega}$ is a family of regular measures for the conditional expectation $E\left[\cdot \mid \mathcal{B}_{0} \otimes \mathcal{F}_{0}\right]$ with respect to $\mathcal{B}_{0} \otimes \mathcal{F}_{0}$ and $\lambda \times \mathbb{P}$. 
Proof. Given any function $f=f(\theta, \omega) \in L_{\lambda \times \mathbb{P}}^{1}$

$$
E\left[f \mid \mathcal{B}_{0} \otimes \mathcal{F}_{0}\right]=E\left[E\left[f \mid \mathcal{B} \otimes \mathcal{F}_{0}\right] \mid \mathcal{B}_{0} \otimes \mathcal{F}_{0}\right]=E\left[\tilde{f} \mid \mathcal{B}_{0} \otimes \mathcal{F}_{0}\right]
$$

where $\tilde{f}$ is the function specified by Lemma A.11. A second application of this lemma with $\mathcal{B} \otimes \mathcal{F}_{0}$ in the role of $\mathcal{B} \otimes \mathcal{F}$ and with $\mathcal{B}_{0}$ in the role of $\mathcal{F}_{0}$ gives that

$$
(\theta, \omega) \mapsto \int_{\Theta} \int_{\Omega} f(x, z) d \mathbb{P}_{\omega}(z) d \lambda_{\theta}(x)
$$

defines a $\mathcal{B}_{0} \otimes \mathcal{F}_{0}$-measurable version of $E\left[f \mid \mathcal{B}_{0} \otimes \mathcal{F}_{0}\right]$.

We finish this section by stating two widely known limit theorems, which are building blocks of the results presented in this paper.

Theorem A.13 (The Lindeberg-Lévy theorem for martingales). For each $n \in \mathbb{N}^{*}$, let $\Delta_{n 1}, \ldots, \Delta_{n k}, \ldots$ be a sequence of real-valued martingale differences with respect to some increasing filtration $\mathcal{F}_{0}^{n} \subset \cdots \subset \mathcal{F}_{k}^{n} \subset \cdots$.

Define, for every $(n, k) \in \mathbb{N}^{*} \times \mathbb{N}, \sigma_{n k}^{2}:=E\left[\Delta_{n k}^{2} \| \mathcal{F}_{k-1}^{n}\right]$, and assume that $\sum_{k} \Delta_{n k}$ and $\sum_{k} \sigma_{n k}^{2}$ converge with probability one. If for some $\sigma \geq 0$ the following two conditions hold

1. $\sum_{k \geq 1} \sigma_{n k}^{2} \Rightarrow_{n} \sigma^{2}$,

2. $\sum_{k \geq 1} E\left[\Delta_{n k}^{2} I_{\left[\Delta_{n k} \geq \epsilon\right]}\right] \rightarrow_{n} 0$, for every $\epsilon>0$,

then $Z_{n}:=\sum_{k \geq 1} \Delta_{n k} \Rightarrow_{n} \sigma N$ where $N$ is a standard normal random variable.

Proof. [6], p. 477.

Theorem A.14 (The functional form of Theorem A.13). For each $n \in \mathbb{N}^{*}$, let $\Delta_{n 1}, \ldots, \Delta_{n k}, \ldots$ be a sequence of real-valued martingale differences with respect to some increasing filtration $\mathcal{F}_{0}^{n} \subset \cdots \subset \mathcal{F}_{k}^{n} \subset \cdots$.

Define, for every $(n, k) \in \mathbb{N}^{*} \times \mathbb{N}, \sigma_{n k}^{2}:=E\left[\Delta_{n k}^{2} \| \mathcal{F}_{k-1}^{n}\right]$. If for some $\sigma \geq 0$ the following two conditions hold for every $t \geq 0, \epsilon>0$

1. $\sum_{k \leq n t} \sigma_{n k}^{2} \Rightarrow_{n} \sigma^{2} t$,

2. $\sum_{k \leq n t} E\left[\Delta_{n k}^{2} I_{\left[\Delta_{n k} \geq \epsilon\right]}\right] \rightarrow_{n} 0$,

then the random functions $X_{n}(t):=\sum_{k \leq n t} \Delta_{n k}$ converge in distribution, as $n \rightarrow \infty$, to $\sigma W$ in the sense of $D[[0, \infty)]$ where $W$ is a standard Brownian motion.

Proof. This is a slight reformulation of Theorem 18.2 in [7], (pp. 194-195): the case $\sigma>0$ follows by a simple renormalization, and to cover the case $\sigma=0$, note that the convergence (18.6) in [7] becomes a simple consequence of the definition given there of $\zeta_{n k}$ and the hypothesis (corresponding to $\sigma=0$ )

$$
\sum_{k \leq n t} \sigma_{n k}^{2} \Rightarrow_{n} 0
$$

for every $t \geq 0$. 


\section{Acknowledgements}

This work is part of the research project carried out by the author under the tutelage of M. Peligrad, and he is indebted to her for suggesting accurate references and giving important comments, in particular with regards to the scope of our methods under the weakened Hannan condition (37).

My conversations with C. Dragan were very useful. Inspirational suggestions were given by professors W. Bryc and Y. Wang during our meetings at the University of Cincinnati Probability Seminar.

It is also difficult to overestimate the role of the anonymous referee with regards to improving the presentation and the results presented along this paper. Besides many stylistic corrections, the author would like to acknowledge that the referee's suggestions gave rise to the ideas leading to the proof of Theorem 3 under (37) presented here ${ }^{19}$ and the corresponding result under condition (39). The referee also suggested to look at (42) as a suitable condition for the $\lambda$-a.e. fulfillment of (39), providing a reference ([2]) that led to the materialization of Proposition 5.12 and its consequences.

The author was supported by the NSF Grants DMS-1208237, DMS-1512936, and by a Laws scholarship for the majority of the time of research and writing of this paper. The revision following the last report by the referee was done during the first two months of the author's stay at the École Polytechnique, supported by a grant from the Risk Foundation.

\section{References}

[1] Akcoglu, M.A. and Sucheston, L. (1978). A ratio ergodic theorem for superadditive processes. Z. Wahrsch. Verw. Gebiete 44 269-278. MR0509202

[2] Assani, I. (1990). Minimal convergence on $L^{p}$ spaces. Ergodic Theory Dynam. Systems $10411-420$. MR1074311

[3] Barrera, D. (2015). An example of non-quenched convergence in the conditional CLT for discrete Fourier transforms. ALEA Lat. Am. J. Probab. Math. Stat. 12 685-697. MR3430503

[4] Barrera, D. (2016). Quenched Asymptotics for the Discrete Fourier Transforms of a Stationary Process Doctoral Dissertation. Version May 2016. Available at arXiv:1604.00428v2.

[5] Barrera, D. and Peligrad, M. (2016). Quenched limit theorems for Fourier transforms and periodogram. Bernoulli 22 275-301. MR3449783

[6] Billingsley, P. (1995). Probability and Measure, 3rd ed. Wiley Series in Probability and Mathematical Statistics. New York: Wiley. MR1324786

[7] Billingsley, P. (1999). Convergence of Probability Measures, 2nd ed. Wiley Series in Probability and Statistics: Probability and Statistics. New York: Wiley. MR1700749

[8] Cuny, C. and Merlevède, F. (2014). On martingale approximations and the quenched weak invariance principle. Ann. Probab. 42 760-793. MR3178473

[9] Cuny, C., Merlevède, F. and Peligrad, M. (2013). Law of the iterated logarithm for the periodogram. Stochastic Process. Appl. 123 4065-4089. MR3091099

[10] Cuny, C. and Volný, D. (2013). A quenched invariance principle for stationary processes. ALEA Lat. Am. J. Probab. Math. Stat. 10 107-115. MR3083921

\footnotetext{
${ }^{19}$ The previous proof departed from a more elementary level but it was also longer, the reader can consult it in [4].
} 
[11] Dedecker, J., Merlevède, F. and Peligrad, M. (2014). A quenched weak invariance principle. Ann. I.H.P. Probab. Stat. 50 872-898. MR3224292

[12] Dehling, H., Durieu, O. and Volny, D. (2009). New techniques for empirical processes of dependent data. Stochastic Process. Appl. 119 3699-3718. MR2568292

[13] Durieu, O. (2009). Independence of four projective criteria for the weak invariance principle. ALEA Lat. Am. J. Probab. Math. Stat. 5 21-26. MR2475604

[14] Durrett, R. (2010). Probability: Theory and Examples, 4th ed. Cambridge Series in Statistical and Probabilistic Mathematics. Cambridge: Cambridge Univ. Press. MR2722836

[15] Einsiedler, M. and Ward, T. (2011). Ergodic Theory with a View Towards Number Theory. Graduate Texts in Mathematics 259. London: Springer. MR2723325

[16] Eisner, T., Farkas, B., Haase, M. and Nagel, R. (2015). Operator Theoretic Aspects of Ergodic Theory. Graduate Texts in Mathematics 272. New York: Springer. MR3410920

[17] Hunt, R.A. and Young, W.S. (1974). A weighted norm inequality for Fourier series. Bull. Amer. Math. Soc. 80 274-277. MR0338655

[18] Krengel, U. (1985). Ergodic Theorems. de Gruyter Studies in Mathematics 6. Berlin: de Gruyter. MR0797411

[19] Lifshits, M.A. and Peligrad, M. (2015). On the spectral density of stationary processes and random fields. Zap. Nauchn. Sem. S.-Peterburg. Otdel. Mat. Inst. Steklov. (POMI) 441 274-285. MR3504510

[20] Peligrad, M. and Wu, W.B. (2010). Central limit theorem for Fourier transforms of stationary processes. Ann. Probab. 38 2009-2022. MR2722793

[21] Quas, A. (2009). Ergodicity and mixing properties. In Encyclopedia of Complexity and Systems Science 2918-2933.

[22] Revu, D. and Yor, M. (2005). Continuous Martingales and Brownian Motion, Grundlehren Math. Wiss. New York: Springer. Corrected 3rd printing.

[23] Wu, W.B. (2005). Fourier transforms of stationary processes. Proc. Amer. Math. Soc. 133 285-293 (electronic). MR2086221

Received June 2015 and revised September 2016 